\title{
Rigidity of the canonical isometric imbedding of the Cayley projective plane $P^{2}(\mathrm{Cay})$
}

\author{
Yoshio AgAOKA and Eiji KANEDA
}

(Received April 30, 2003)

\begin{abstract}
In [7], we have proved that $P^{2}(\boldsymbol{C a y})$ cannot be isometrically immersed into $\boldsymbol{R}^{25}$ even locally. In this paper, we investigate isometric immersions of $P^{2}(\boldsymbol{C a y})$ into $\boldsymbol{R}^{26}$ and prove that the canonical isometric imbedding $\boldsymbol{f}_{0}$ of $P^{2}(\boldsymbol{C a y})$ into $\boldsymbol{R}^{26}$, which is defined in Kobayashi [17], is rigid in the following strongest sense: Any isometric immersion $\boldsymbol{f}_{1}$ of a connected open set $U\left(\subset P^{2}(\boldsymbol{C a} \boldsymbol{y})\right)$ into $\boldsymbol{R}^{26}$ coincides with $\boldsymbol{f}_{0}$ up to a euclidean transformation of $\boldsymbol{R}^{26}$, i.e., there is a euclidean transformation $a$ of $\boldsymbol{R}^{26}$ satisfying $\boldsymbol{f}_{1}=a \boldsymbol{f}_{0}$ on $U$.

Key words: curvature invariant, isometric immersion, Cayley projective plane, rigidity.
\end{abstract}

\section{Introduction}

In the previous paper [7], we investigated the problem of (local) isometric immersions of the quaternion projective plane $P^{2}(\boldsymbol{H})$ and the Cayley projective plane $P^{2}(\boldsymbol{C a y})$. In particular, we proved the following nonexistence theorem of (local) isometric immersions:

Theorem 1 Any open set of the Cayley projective plane $P^{2}(\boldsymbol{C a y})$ cannot be isometrically immersed into $\boldsymbol{R}^{25}$.

As is well-known, there is an isometric immersion $\boldsymbol{f}_{0}$ of $P^{2}(\boldsymbol{C a y})$ into the euclidean space $\boldsymbol{R}^{26}$, which is called the canonical isometric imbedding of $P^{2}(\boldsymbol{C a y})$ (Kobayashi [17]). This fact, together with Theorem 1, implies that $\boldsymbol{R}^{26}$ is the least dimensional euclidean space into which $P^{2}(\boldsymbol{C a y})$ can be (locally) isometrically immersed.

In this paper, we consider (local) isometric immersions of $P^{2}(\boldsymbol{C a y})$ into $\boldsymbol{R}^{26}$ and discuss the rigidity of the canonical isometric imbedding $\boldsymbol{f}_{0}$. Concerning the rigidity of $\boldsymbol{f}_{0}$ Kaneda [15] has shown that the canonical isometric imbedding $\boldsymbol{f}_{0}$ is of finite type, i.e., the space of local infinitesimal isometric deformations of $\boldsymbol{f}_{0}$ is of finite dimension. However, it seems to the authors that any further result concerning the rigidity of $\boldsymbol{f}_{0}$ has not been

2000 Mathematics Subject Classification : 17B20, 53B25, 53C24, 53C35. 
obtained.

In the present paper, we will show the rigidity of the canonical isometric imbedding $\boldsymbol{f}_{0}$ in the following strongest form:

Theorem 2 Let $\boldsymbol{f}_{0}$ be the canonical isometric imbedding of $P^{2}(\boldsymbol{C a y})$ into the euclidean space $\boldsymbol{R}^{26}$. Then, for any isometric immersion $\boldsymbol{f}_{1}$ defined on a connected open set $U$ of $P^{2}(\boldsymbol{C a y})$ into $\boldsymbol{R}^{26}$, there exists a euclidean transformation a of $\boldsymbol{R}^{26}$ satisfying $\boldsymbol{f}_{1}=a \boldsymbol{f}_{0}$ on $U$.

To prove Theorem 2, we first establish a rigidity theorem for an isometric immersion of a Riemannian manifold. Let $M$ be an $n$-dimensional Riemannian manifold and let $\boldsymbol{f}_{0}$ be an isometric immersion of $M$ into the $m$-dimensional euclidean space $\boldsymbol{R}^{m}$. We will prove that if the Gauss equation in codimension $r(=m-n)$ admits essentially one solution everywhere on $M$, then $\boldsymbol{f}_{0}$ is rigid, i.e., for any isometric immersion $\boldsymbol{f}_{1}$ of $M$ into $\boldsymbol{R}^{m}$ there exists a euclidean transformation $a$ of $\boldsymbol{R}^{m}$ such that $\boldsymbol{f}_{1}=a \boldsymbol{f}_{0}$ (see Theorem 5). This theorem may be established by various methods; for example, by combining the results of Nomizu [19] and Szczarba [21], [22] (cf. Agaoka [1]) or by solving a differential system of Pfaff (cf. BishopCrittenden [10], Ch. X). In this paper, we will give a simple proof based on a congruence theorem of differentiable mappings, which is easy to understand and gives a clear view on the geometric meaning (see Theorem 6).

Next, we will show that for the Cayley projective plane $P^{2}(\boldsymbol{C a y})$ the Gauss equation in codimension $10\left(=26-\operatorname{dim} P^{2}(\boldsymbol{C a y})\right)$ admits essentially one solution (see Theorem 10). To show this, we utilize the results obtained in [6] and [7]. Among all, the result concerning pseudo-abelian subspaces (Proposition 8) plays an important role in our proof.

Then, Theorem 2 is a direct consequence of Theorem 5 and Theorem 10.

Throughout this paper we assume the differentiability of class $C^{\infty}$. Notations for Lie algebras are the same as those used in [6] and [7].

\section{The Gauss equation}

Let $M$ be a Riemannian manifold and $T(M)$ the tangent bundle of $M$. We denote by $g$ the Riemannian metric of $M$ and by $R$ the Riemannian curvature tensor of type $(1,3)$ with respect to $g$.

Let $\boldsymbol{N}$ be a euclidean vector space, i.e., $\boldsymbol{N}$ is a vector space over $\boldsymbol{R}$ endowed with an inner product $\langle$,$\rangle . Let p \in M$ and let $S^{2} T_{p}^{*}(M) \otimes \boldsymbol{N}$ be the space of $\boldsymbol{N}$-valued symmetric bilinear forms on $T_{p}(M)$. We call the 
following equation on $\boldsymbol{\Psi} \in S^{2} T_{p}^{*}(M) \otimes \boldsymbol{N}$ the Gauss equation at $p \in M$ :

$$
-g_{p}\left(R_{p}(x, y) z, w\right)=\langle\boldsymbol{\Psi}(x, z), \boldsymbol{\Psi}(y, w)\rangle-\langle\boldsymbol{\Psi}(x, w), \boldsymbol{\Psi}(y, z)\rangle,
$$

where $x, y, z, w \in T_{p}(M)$. We denote by $\mathcal{G}_{p}(\boldsymbol{N})$ the set of all solutions of (2.1), which is called the Gaussian variety associated with $\boldsymbol{N}$ at $p \in M$. As is well-known, $\mathcal{G}_{p}(\boldsymbol{N})=\emptyset$ happens in case the dimensionality $r(=\operatorname{dim} \boldsymbol{N})$ is so small, however, $\mathcal{G}_{p}(\boldsymbol{N}) \neq \emptyset$ if $r$ is sufficiently large (see Cartan [11] or Kaneda-Tanaka [16]).

Let $\boldsymbol{N}_{1}$ and $\boldsymbol{N}_{2}$ be two euclidean vector spaces and let $\varphi$ be a linear mapping of $\boldsymbol{N}_{1}$ to $\boldsymbol{N}_{2}$. Define a linear map $\widehat{\varphi}$ of $S^{2} T_{p}^{*}(M) \otimes \boldsymbol{N}_{1}$ to $S^{2} T_{p}^{*}(M) \otimes N_{2}$ by

$$
(\widehat{\varphi} \boldsymbol{\Psi})(x, y)=\varphi(\boldsymbol{\Psi}(x, y)), \boldsymbol{\Psi} \in S^{2} T_{p}^{*}(M) \otimes \boldsymbol{N}_{1}, x, y \in T_{p}(M) .
$$

Then, we can easily verify

Lemma 3 Let $\varphi$ be a linear mapping of a euclidean vector space $\boldsymbol{N}_{1}$ to a euclidean vector space $\boldsymbol{N}_{2}$. Assume that $\varphi$ is isometric, i.e., $\langle\varphi(x), \varphi(y)\rangle_{2}$ $=\langle x, y\rangle_{1}\left(x, y \in \boldsymbol{N}_{1}\right)$, where $\langle,\rangle_{i}(i=1,2)$ denotes the inner product of $\boldsymbol{N}_{i}$. Then $\widehat{\varphi} \mathcal{G}_{p}\left(\boldsymbol{N}_{1}\right) \subset \mathcal{G}_{p}\left(\boldsymbol{N}_{2}\right)$. In particular, if $\operatorname{dim} \boldsymbol{N}_{1}=\operatorname{dim} \boldsymbol{N}_{2}$, then $\widehat{\varphi} \mathcal{G}_{p}\left(\boldsymbol{N}_{1}\right)=\mathcal{G}_{p}\left(\boldsymbol{N}_{2}\right)$.

In view of Lemma 3, the solvability of the Gauss equation (2.1) substantially depends on the dimensionality of $\boldsymbol{N}$. To emphasize $\operatorname{dim} \boldsymbol{N}$ we call (2.1) the Gauss equation in codimension $r(=\operatorname{dim} \boldsymbol{N})$.

Let $\boldsymbol{N}$ be a euclidean vector space and let $O(\boldsymbol{N})$ be the orthogonal transformation group of $\boldsymbol{N}$. We define an action of $O(\boldsymbol{N})$ on $S^{2} T_{p}^{*}(M) \otimes \boldsymbol{N}$ by

$$
(h \boldsymbol{\Psi})(x, y)=h(\boldsymbol{\Psi}(x, y)),
$$

where $\boldsymbol{\Psi} \in S^{2} T_{p}^{*}(M) \otimes \boldsymbol{N}, h \in O(\boldsymbol{N}), x, y \in T_{p}(M)$. We say that two elements $\boldsymbol{\Psi}$ and $\boldsymbol{\Psi}^{\prime} \in S^{2} T_{p}^{*}(M) \otimes \boldsymbol{N}$ are equivalent if there is an element $h \in O(\boldsymbol{N})$ such that $\boldsymbol{\Psi}^{\prime}=h \boldsymbol{\Psi}$. It is easily seen that if $\boldsymbol{\Psi}$ and $\boldsymbol{\Psi}^{\prime} \in$ $S^{2} T_{p}^{*}(M) \otimes \boldsymbol{N}$ are equivalent and $\boldsymbol{\Psi} \in \mathcal{G}_{p}(\boldsymbol{N})$, then $\boldsymbol{\Psi}^{\prime} \in \mathcal{G}_{p}(\boldsymbol{N})$. We say that the Gaussian variety $\mathcal{G}_{p}(\boldsymbol{N})$ is $E O S$ if $\mathcal{G}_{p}(\boldsymbol{N}) \neq \emptyset$ and if it consists of essentially one solution, i.e., any solutions of the Gauss equation (2.1) are equivalent to each other under the action of $O(\boldsymbol{N})$. 
Proposition 4 Let $M$ be a Riemannian manifold and let $p \in M$. Let $\mathbf{N}$ be an $r$-dimensional euclidean vector space such that $\mathcal{G}_{p}(\boldsymbol{N})$ is EOS. Then:

(1) Let $\boldsymbol{\Psi}$ be an arbitrary element of $\mathcal{G}_{p}(\boldsymbol{N})$. Then, the vectors $\boldsymbol{\Psi}(x, y)$

$\left(x, y \in T_{p}(M)\right)$ span the whole space $\boldsymbol{N}$.

(2) Let $\boldsymbol{N}_{1}$ be a euclidean vector space. Then:

(2a) $\mathcal{G}_{p}\left(\boldsymbol{N}_{1}\right)=\emptyset$ if $\operatorname{dim} \boldsymbol{N}_{1}<r$

(2b) $\mathcal{G}_{p}\left(\boldsymbol{N}_{1}\right)$ is EOS if $\operatorname{dim} \boldsymbol{N}_{1}=r$;

(2c) $\mathcal{G}_{p}\left(\boldsymbol{N}_{1}\right)$ is not EOS if $\operatorname{dim} \boldsymbol{N}_{1}>r$.

Proof. Note that if $\boldsymbol{\Psi}^{\prime} \in S^{2} T_{p}^{*}(M) \otimes \boldsymbol{N}$ is equivalent to $\boldsymbol{\Psi}$, then we have $\left|\boldsymbol{\Psi}^{\prime}(x, y)\right|=|\boldsymbol{\Psi}(x, y)|$ for any $x, y \in T_{p}(M)$, where $|\boldsymbol{n}|$ denotes the norm of $\boldsymbol{n} \in \boldsymbol{N}$ with respect to $\langle$,$\rangle .$

Now, suppose that the vectors $\boldsymbol{\Psi}(x, y)\left(x, y \in T_{p}(M)\right)$ do not span the whole space $\boldsymbol{N}$. Then, there is a non-zero vector $\boldsymbol{n} \in \boldsymbol{N}$ satisfying $\langle\boldsymbol{n}, \boldsymbol{\Psi}(x, y)\rangle=0$ for any $x, y \in T_{p}(M)$. Define an element $\boldsymbol{\Psi}^{\prime} \in S^{2} T_{p}^{*}(M) \otimes$ $N$ by

$$
\boldsymbol{\Psi}^{\prime}=\boldsymbol{\Psi}+\left(\xi^{*}\right)^{2} \otimes \boldsymbol{n},
$$

where $\xi^{*}$ is a non-zero element of $T_{p}^{*}(M)$. Then, it is easy to see that $\boldsymbol{\Psi}^{\prime} \in \mathcal{G}_{p}(\boldsymbol{N})$. However, by a simple calculation, we have $\left|\boldsymbol{\Psi}^{\prime}(x, x)\right|^{2}=$ $|\boldsymbol{\Psi}(x, x)|^{2}+|\boldsymbol{n}|^{2} \xi^{*}(x)^{2}$. Therefore, if we take $x \in T_{p}(M)$ such that $\xi^{*}(x) \neq$ 0 , then we have $\left|\Psi^{\prime}(x, x)\right| \neq|\Psi(x, x)|$. This proves that $\boldsymbol{\Psi}^{\prime}$ is not equivalent to $\boldsymbol{\Psi}$ and hence $\mathcal{G}_{p}(\boldsymbol{N})$ is not EOS. Thus, we obtain (1).

Next we prove (2). First assume $\operatorname{dim} \boldsymbol{N}_{1}=r$. Let $\varphi$ be an isometric linear isomorphism of $\boldsymbol{N}$ onto $\boldsymbol{N}_{1}$. Then we have $O\left(\boldsymbol{N}_{1}\right)=\varphi \cdot O(\boldsymbol{N}) \cdot \varphi^{-1}$. Moreover, by Lemma 3 we have $\widehat{\varphi} \mathcal{G}_{p}(\boldsymbol{N})=\mathcal{G}_{p}\left(\boldsymbol{N}_{1}\right)$. Since $\mathcal{G}_{p}(\boldsymbol{N})$ is EOS, $O(\boldsymbol{N})$ acts transitively on $\mathcal{G}_{p}(\boldsymbol{N})$. Therefore, it is easily seen that $O\left(\boldsymbol{N}_{1}\right)$ acts transitively on $\mathcal{G}_{p}\left(\boldsymbol{N}_{1}\right)$. This proves that $\mathcal{G}_{p}\left(\boldsymbol{N}_{1}\right)$ is EOS.

We next consider the case $\operatorname{dim} \boldsymbol{N}_{1}<r$. Suppose that $\mathcal{G}_{p}\left(\boldsymbol{N}_{1}\right) \neq \emptyset$ and $\boldsymbol{\Psi}_{1} \in \mathcal{G}_{p}\left(\boldsymbol{N}_{1}\right)$. Let $\varphi$ be an isometric linear mapping of $\boldsymbol{N}_{1}$ to $\boldsymbol{N}$. Then, we know that $\widehat{\varphi} \boldsymbol{\Psi}_{1} \in \mathcal{G}_{p}(\boldsymbol{N})$ and the vectors $\left(\widehat{\varphi} \boldsymbol{\Psi}_{1}\right)(x, y)\left(x, y \in T_{p}(M)\right)$ are contained in the proper subspace $\varphi\left(\boldsymbol{N}_{1}\right)(\subsetneq \boldsymbol{N})$. This contradicts (1). The case $\operatorname{dim} \boldsymbol{N}_{1}>r$ is similarly dealt with.

We say that a Riemannian manifold $M$ is formally rigid in codimension $r$ if there is a euclidean vector space $\boldsymbol{N}$ with $\operatorname{dim} \boldsymbol{N}=r$ such that the Gaussian variety $\mathcal{G}_{p}(\boldsymbol{N})$ is EOS at each $p \in M$. By virtue of Proposition 4 (2), we know that if $M$ is formally rigid in codimension $r$, then it is not formally 
rigid in any other codimension $r^{\prime}(\neq r)$.

Remark 1 It should be noted that there is a Riemannian manifold $M$ that is not formally rigid in any codimension $r$. For example, assume that $M$ is the space of negative constant curvature of dimension $n$. Let $N$ be a euclidean vector space of dimension $r$. Then, by Ôtsuki's lemma we have $\mathcal{G}_{p}(\boldsymbol{N})=\emptyset$ if $r<n-1$ (see Ôtsuki [20]). On the other hand, Kaneda [13] proved that if $r=n-1$, then $\mathcal{G}_{p}(\boldsymbol{N}) \neq \emptyset$ and around a suitable $\boldsymbol{\Psi}_{0} \in$ $\mathcal{G}_{p}(\boldsymbol{N}), \mathcal{G}_{p}(\boldsymbol{N})$ forms a submanifold of $S^{2} T_{p}^{*}(M) \otimes \boldsymbol{N}$ of dimension $n(n-1)$ (see Theorem 3.1 of [13]). Since $n(n-1)>\operatorname{dim} O(\boldsymbol{N}), \mathcal{G}_{p}(\boldsymbol{N})$ cannot be EOS. If $r \geq n$, then by Proposition $4(2 a)$ we know that $\mathcal{G}_{p}(\boldsymbol{N})$ is not EOS. Accordingly, the space of negative constant curvature $M$ is not formally rigid in any codimension $r$.

Remark 2 For each Riemannian submanifold $M \subset \boldsymbol{R}^{m}$ listed below, $\mathcal{G}_{p}(\boldsymbol{N})$ is known to be EOS at each $p \in M$, where $\boldsymbol{N}$ is the normal vector space of $M$ at $p$ in $\boldsymbol{R}^{m}$ :

(1) The sphere $S^{n} \subset \boldsymbol{R}^{n+1}(n \geq 3)$;

(2) The symplectic group $S p(2) \subset \boldsymbol{R}^{16}$ (see Agaoka [1]);

(3) A submanifold $M \subset \boldsymbol{R}^{m}$ with type number $\geq 3$ (see Allendoerfer [9], Kobayashi-Nomizu [18]).

Consequently, these submanifolds are formally rigid in our sense and it has been proved that they are actually rigid in $\boldsymbol{R}^{m}$ (see [1], [9]).

However, we note that the formal rigidness of $M$ in codimension $r$ does not imply the existence of an isometric immersion of $M$ into $\boldsymbol{R}^{n+r}(n=$ $\operatorname{dim} M)$. Indeed, Kaneda [14] gave an example of three dimensional Riemannian manifold $M$ that is formally rigid in codimension 1 but cannot be locally isometrically immersed into $\boldsymbol{R}^{4}$.

We will prove in the next section that if a connected Riemannian manifold $M$ is formally rigid in codimension $r$ and if there is an isometric immersion $\boldsymbol{f}$ of $M$ into $\boldsymbol{R}^{n+r}(n=\operatorname{dim} M)$, then $M$ (precisely, $\left.\boldsymbol{f}(M)\right)$ is actually rigid in $\boldsymbol{R}^{n+r}$ (see Theorem 5).

\section{Rigidity theorem}

In this section, we will prove the following rigidity theorem:

Theorem 5 Let $M$ be an n-dimensional Riemannian manifold and let $\boldsymbol{f}_{0}$ be an isometric immersion of $M$ into the euclidean space $\boldsymbol{R}^{m}$. Assume: 
(1) $M$ is connected;

(2) $M$ is formally rigid in codimension $r=m-n$.

Then, any isometric immersion $\boldsymbol{f}_{1}$ of $M$ into the euclidean space $\boldsymbol{R}^{m}$ coincides with $\boldsymbol{f}_{0}$ up to a euclidean transformation of $\boldsymbol{R}^{m}$, i.e., there exists a euclidean transformation a of $\boldsymbol{R}^{m}$ such that $\boldsymbol{f}_{1}=a \boldsymbol{f}_{0}$.

Before proceeding to the proof of Theorem 5, we make some preparations. Let $M\left(m, m^{\prime}\right)$ be the space of real matrices of degree $m \times m^{\prime}$, where $m$ and $m^{\prime}$ are non-negative integers. In what follows we identify $M(m, 1)$ with the $m$-dimensional euclidean space $\boldsymbol{R}^{m}$ in a natural way. Then, we note that the canonical inner product $\langle$, $\rangle$ of $\boldsymbol{R}^{m}$ is given by $\langle\boldsymbol{v}, \boldsymbol{w}\rangle={ }^{t} \boldsymbol{v}$. $\boldsymbol{w}$ for $\boldsymbol{v}, \boldsymbol{w} \in \boldsymbol{R}^{m}$.

Let us define an operation of $M(m, m)$ on $\boldsymbol{R}^{m}$ by

$$
M(m, m) \times \boldsymbol{R}^{m} \ni(H, \boldsymbol{v}) \longmapsto H \cdot \boldsymbol{v} \in \boldsymbol{R}^{m},
$$

where - means the usual matrix multiplication.

Let $\nabla$ be the Riemannian connection associated with $M$. Let $\boldsymbol{f}=$ ${ }^{t}\left(f^{1}, \ldots, f^{m}\right)$ be a differentiable map of $M$ into the euclidean space $\boldsymbol{R}^{m}$. By $\overbrace{\nabla \cdots \nabla}^{k} \boldsymbol{f}$ we denote the $k$-th order covariant derivative of $\boldsymbol{f}$, which is defined as follows:

$$
\overbrace{\nabla_{x_{1}} \cdots \nabla_{x_{k}}}^{k} \boldsymbol{f}={ }^{t}(\ldots, \overbrace{\nabla_{x_{1}} \cdots \nabla_{x_{k}}}^{k} f^{i}, \ldots) \in \boldsymbol{R}^{m},
$$

where $p \in M ; x_{1}, \ldots, x_{k} \in T_{p}(M)$. (Precisely, see Tanaka [23], KanedaTanaka [16] or Kaneda [14].) It is known that $\nabla \nabla \boldsymbol{f}$ and $\nabla \nabla \nabla \boldsymbol{f}$ satisfy the following integrability conditions:

$$
\begin{aligned}
\nabla_{x} \nabla_{y} \boldsymbol{f} & =\nabla_{y} \nabla_{x} \boldsymbol{f}, \\
\nabla_{z} \nabla_{x} \nabla_{y} \boldsymbol{f} & =\nabla_{x} \nabla_{z} \nabla_{y} \boldsymbol{f}-\nabla_{R(z, x) y} \boldsymbol{f} .
\end{aligned}
$$

We say that a differentiable map $\boldsymbol{f}$ of $M$ into $\boldsymbol{R}^{m}$ is 2-generic if at each $p \in M$, the whole space $\boldsymbol{R}^{m}$ is spanned by the vectors of the form $\nabla_{x} \boldsymbol{f}\left(x \in T_{p}(M)\right), \nabla_{y} \nabla_{z} \boldsymbol{f}\left(y, z \in T_{p}(M)\right)$. It is clear that if $\boldsymbol{f}$ is 2-generic, then we have the inequality $m \leq(1 / 2) n(n+3)$. Note that a 2-generic map $f$ is not necessarily an immersion.

We first show the following congruence theorem: 
Theorem 6 Let $M$ be an n-dimensional Riemannian manifold and let $\boldsymbol{f}_{i}(i=0,1)$ be two differentiable maps of $M$ into the euclidean space $\boldsymbol{R}^{m}$. Assume:

(1) $M$ is connected;

(2) $\boldsymbol{f}_{0}$ is 2-generic;

(3) At each $p \in M$ there is an element $H(p) \in O(m)$ satisfying

$$
\begin{aligned}
\nabla_{x} \boldsymbol{f}_{1} & =H(p) \cdot\left(\nabla_{x} \boldsymbol{f}_{0}\right), & & \forall x \in T_{p}(M), \\
\nabla_{y} \nabla_{z} \boldsymbol{f}_{1} & =H(p) \cdot\left(\nabla_{y} \nabla_{z} \boldsymbol{f}_{0}\right), & & \forall y, z \in T_{p}(M) .
\end{aligned}
$$

Then, $\boldsymbol{f}_{1}$ coincides with $\boldsymbol{f}_{0}$ up to a euclidean transformation of $\boldsymbol{R}^{m}$. More precisely, $H(p)$ is identically equal to a constant value $H_{0} \in O(m)$ everywhere on $M$ and $\boldsymbol{f}_{1}$ can be written as $\boldsymbol{f}_{1}=H_{0} \boldsymbol{f}_{0}+\boldsymbol{c}_{0}$, where $\boldsymbol{c}_{0}$ is a constant vector of $\boldsymbol{R}^{m}$.

Proof. We first note that, since $\boldsymbol{f}_{0}$ is 2-generic, $H(p)$ satisfying (3.3) and (3.4) is uniquely determined at each $p \in M$ and the map $H: M \ni$ $p \longmapsto H(p) \in O(m)$ is differentiable. Via the canonical inclusion $O(m) \subset$ $M(m, m)$, we can regard $H$ as an $M(m, m)$-valued function on $M$ satisfying

$$
{ }^{t} H H=I_{m},
$$

where $I_{m}$ denotes the identity matrix of degree $m$. Differentiate (3.5) covariantly. Then by Leibnitz' law we get

$$
\nabla_{x}\left({ }^{t} H\right) H(p)+{ }^{t} H(p)\left(\nabla_{x} H\right)=0, \quad \forall x \in T_{p}(M) .
$$

In this equality, the covariant derivative $\nabla_{x} H$ means the element of $M(m, m)$ given by $\nabla_{x} H=\left(\nabla_{x} h_{i}^{j}\right)$, where $h_{i}^{j}$ denotes the $(i, j)$-component of $H$. By the very definition of $\nabla_{x} H$ we have $\nabla_{x}\left({ }^{t} H\right)={ }^{t}\left(\nabla_{x} H\right)$.

Let us define an $M(m, m)$-valued 1-form $L$ by

$$
L(x)={ }^{t} H(p)\left(\nabla_{x} H\right), \quad x \in T_{p}(M) .
$$

Then, by (3.6) we have

$$
{ }^{t} L(x)+L(x)=0, \quad \forall x \in T_{p}(M),
$$

implying that the matrix $L(x) \in M(m, m)$ is skew-symmetric.

We now show that the equality $L(x)=0$ holds for any $x \in T_{p}(M)$. Since $\boldsymbol{f}_{0}$ is 2 -generic, it suffices to prove

$$
L(y) \cdot\left(\nabla_{x} \boldsymbol{f}_{0}\right)=0, \quad \forall x, y \in T_{p}(M),
$$




$$
L(z) \cdot\left(\nabla_{y} \nabla_{x} \boldsymbol{f}_{0}\right)=0, \quad \forall x, y, z \in T_{p}(M) .
$$

Differentiating (3.3) and (3.4) covariantly, we have

$$
\begin{array}{r}
\nabla_{y} \nabla_{x} \boldsymbol{f}_{1}=\nabla_{y} H \cdot\left(\nabla_{x} \boldsymbol{f}_{0}\right)+H(p) \cdot\left(\nabla_{y} \nabla_{x} \boldsymbol{f}_{0}\right), \\
\forall x, y \in T_{p}(M), \\
\nabla_{z} \nabla_{y} \nabla_{x} \boldsymbol{f}_{1}=\nabla_{z} H \cdot\left(\nabla_{y} \nabla_{x} \boldsymbol{f}_{0}\right)+H(p) \cdot\left(\nabla_{z} \nabla_{y} \nabla_{x} \boldsymbol{f}_{0}\right), \\
\forall x, y, z \in T_{p}(M) .
\end{array}
$$

Then by (3.4) and (3.11) we have $\nabla_{y} H \cdot\left(\nabla_{x} \boldsymbol{f}_{0}\right)=0$ for each $x, y \in T_{p}(M)$. Consequently, multiplying ${ }^{t} H(p)$ from the left, we have (3.9).

We now prove (3.10). Exchanging $z$ and $y$ in (3.12), we have

$$
\begin{aligned}
& \nabla_{y} \nabla_{z} \nabla_{x} \boldsymbol{f}_{1}=\nabla_{y} H \cdot\left(\nabla_{z} \nabla_{x} \boldsymbol{f}_{0}\right)+H(p) \cdot\left(\nabla_{y} \nabla_{z} \nabla_{x} \boldsymbol{f}_{0}\right), \\
& \forall x, y, z \in T_{p}(M) .
\end{aligned}
$$

Subtract (3.13) from (3.12). Then, using the integrability condition (3.2) and the equality (3.3), we have

$$
\nabla_{z} H\left(\nabla_{y} \nabla_{x} \boldsymbol{f}_{0}\right)=\nabla_{y} H\left(\nabla_{z} \nabla_{x} \boldsymbol{f}_{0}\right), \quad \forall x, y, z \in T_{p}(M) .
$$

Consequently, multiplying ${ }^{t} H(p)$ from the left, we get

$$
L(z) \cdot\left(\nabla_{y} \nabla_{x} \boldsymbol{f}_{0}\right)=L(y) \cdot\left(\nabla_{z} \nabla_{x} \boldsymbol{f}_{0}\right), \quad \forall x, y, z \in T_{p}(M) .
$$

Since $L(z)$ is a skew-symmetric matrix, we have

$$
\left\langle L(z) \cdot\left(\nabla_{y} \nabla_{x} \boldsymbol{f}_{0}\right), \nabla_{u} \boldsymbol{f}_{0}\right\rangle=-\left\langle\nabla_{y} \nabla_{x} \boldsymbol{f}_{0}, L(z) \cdot\left(\nabla_{u} \boldsymbol{f}_{0}\right)\right\rangle=0 .
$$

Therefore, to prove (3.10), we have to show

$$
\left\langle L(z) \cdot\left(\nabla_{y} \nabla_{x} \boldsymbol{f}_{0}\right), \nabla_{v} \nabla_{w} \boldsymbol{f}_{0}\right\rangle=0, \quad \forall x, y, z, v, w \in T_{p}(M) .
$$

Define an element $X \in \otimes^{5} T_{p}^{*}(M)$ by

$$
\begin{array}{r}
X(z, y, x, v, w)=\left\langle L(z) \cdot\left(\nabla_{y} \nabla_{x} \boldsymbol{f}_{0}\right), \nabla_{v} \nabla_{w} \boldsymbol{f}_{0}\right\rangle, \\
x, y, z, v, w \in T_{p}(M) .
\end{array}
$$

In the following, we will show $X(z, y, x, v, w)=0$ for $x, y, z, v, w \in$ $T_{p}(M)$. By the integrability condition (3.1) and by (3.15), we easily know that $X(z, y, x, v, w)$ is symmetric with respect to the pairs $\{x, y\},\{v, w\}$ and $\{z, y\}$. Further, since $L(z)$ is a skew-symmetric endomorphism of $\boldsymbol{R}^{m}$ 
(see (3.8)), it follows that

$$
X(z, y, x, v, w)=-X(z, v, w, y, x) .
$$

Therefore, $X(z, y, x, v, w)$ is anti-symmetric with respect to the pair $\{x, w\}$, because

$$
\begin{aligned}
X(z, y, x, v, w) & =-X(z, v, w, y, x)=-X(v, z, w, y, x) \\
& =X(v, y, x, z, w)=X(y, v, x, z, w) \\
& =-X(y, z, w, v, x)=-X(z, y, w, v, x) .
\end{aligned}
$$

Consequently, we get

$$
\begin{aligned}
X(z, y, x, v, w) & =-X(z, y, w, v, x)=-X(z, w, y, x, v) \\
& =X(z, w, v, x, y)=X(z, v, w, y, x) .
\end{aligned}
$$

This, together with (3.18), proves $X(z, y, x, v, w)=0$. Thus we get (3.10).

By the above argument, we know that $L(x)={ }^{t} H(p)\left(\nabla_{x} H\right)=0$ for any $x \in T_{p}(M)$. This implies that $H$ is a locally constant function and hence $H$ is identically equal to an element $H_{0} \in O(m)$ on $M$, because $M$ is connected. Consequently, the difference $\boldsymbol{c}=\boldsymbol{f}_{1}-H_{0} \cdot \boldsymbol{f}_{0}$ satisfies

$$
\nabla_{x} \boldsymbol{c}=\nabla_{x}\left(\boldsymbol{f}_{1}-H_{0} \cdot \boldsymbol{f}_{0}\right)=\nabla_{x} \boldsymbol{f}_{1}-H_{0} \cdot\left(\nabla_{x} \boldsymbol{f}_{0}\right)=0, \quad \forall x \in T_{p}(M) .
$$

Therefore, $\boldsymbol{c}$ is also identically equal to a constant vector $\boldsymbol{c}_{0} \in \boldsymbol{R}^{m}$, completing the proof of the theorem.

Remark 3 The argument in the proof of the equality $X=0$ is essentially the same that is developed in the proof of the uniqueness of the metric connection of the normal bundle associated with an isometric imbedding (see the proof of Theorem 1 of [19]); It is almost the same that is used to calculate the third prolongation of the symbol of the operator $L$ (see Proposition 2.2 of [16]). Here we remark that $X=0$ can be proved without assuming the existence of (isometric) immersions.

We are now in a position to prove Theorem 5 .

Proof of Theorem 5. We show that the map $\boldsymbol{f}_{i}(i=0,1)$ is 2-generic and for each $p \in M$ there is an element $H(p) \in O(m)$ satisfying the equalities (3.3) and (3.4).

Let $i=0$ or 1 . Let $\boldsymbol{f}_{i *} T_{p}(M)$ (resp. $\boldsymbol{N}_{i}$ ) be the tangent vector space (resp. normal vector space) of $\boldsymbol{f}_{i}(M)$ at $\boldsymbol{f}_{i}(p) \in \boldsymbol{R}^{m}$. Then, we have 
$\operatorname{dim} \boldsymbol{f}_{i *} T_{p}(M)=n$ and $\operatorname{dim} \boldsymbol{N}_{i}=m-n$. We regard $\boldsymbol{f}_{i *} T_{p}(M)$ and $\boldsymbol{N}_{i}$ as euclidean vector spaces endowed with the inner products induced from the inner product $\langle$,$\rangle of \boldsymbol{R}^{m}$. By a natural parallel displacement from $\boldsymbol{f}_{i}(p)$ to the origin $o \in \boldsymbol{R}^{m}$, we regard $\boldsymbol{f}_{i *} T_{p}(M)$ and $\boldsymbol{N}_{i}$ as linear subspaces of $\boldsymbol{R}^{m}$. Since $\boldsymbol{f}_{i}$ is an isometric immersion, $\boldsymbol{f}_{i *} T_{p}(M)$ is spanned by the vectors $\nabla_{x} \boldsymbol{f}_{i}\left(x \in T_{p}(M)\right)$ and

$$
\left\langle\nabla_{x} \boldsymbol{f}_{i}, \nabla_{y} \boldsymbol{f}_{i}\right\rangle=g_{p}(x, y), \quad \forall x, y \in T_{p}(M) .
$$

The second order derivative $\nabla \nabla \boldsymbol{f}_{i}$, which is so called the second fundamental form of $\boldsymbol{f}_{i}$, satisfies $\nabla \nabla \boldsymbol{f}_{i} \in S^{2} T_{p}^{*}(M) \otimes \boldsymbol{N}_{i}$ and $\nabla \nabla \boldsymbol{f}_{i} \in \mathcal{G}_{p}\left(\boldsymbol{N}_{i}\right)$ (see [23], [16]). Since $\mathcal{G}_{p}\left(\boldsymbol{N}_{i}\right)$ is EOS, the vectors $\nabla_{x} \nabla_{y} \boldsymbol{f}_{i}\left(x, y \in T_{p}(M)\right.$ ) span $\boldsymbol{N}_{i}$, implying that $\boldsymbol{f}_{i}$ is 2-generic (see Proposition 4 (1)). Take an isometric linear isomorphism $\varphi_{2}$ of $\boldsymbol{N}_{0}$ onto $\boldsymbol{N}_{1}$. Since $\widehat{\varphi_{2}} \nabla \nabla \boldsymbol{f}_{0} \in \mathcal{G}_{p}\left(\boldsymbol{N}_{1}\right)$ and since $\mathcal{G}_{p}\left(\boldsymbol{N}_{1}\right)$ is EOS (see Proposition $4(2 b)$ ), there is an element $h_{1} \in$ $O\left(\boldsymbol{N}_{1}\right)$ such that $h_{1}\left(\widehat{\varphi_{2}} \nabla \nabla \boldsymbol{f}_{0}\right)=\nabla \nabla \boldsymbol{f}_{1}$. On the other hand, in view of (3.19) we also know that there is an isometric linear isomorphism $\varphi_{1}$ of $\boldsymbol{f}_{0 *} T_{p}(M)$ onto $\boldsymbol{f}_{1 *} T_{p}(M)$ satisfying $\varphi_{1}\left(\nabla_{x} \boldsymbol{f}_{0}\right)=\nabla_{x} \boldsymbol{f}_{1}\left(x \in T_{p}(M)\right)$. Define a linear endomorphism $H(p)$ of $\boldsymbol{R}^{m}$ satisfying $\left.H(p)\right|_{\boldsymbol{f}_{0 *} T_{p}(M)}=\varphi_{1}$ and $\left.H(p)\right|_{\boldsymbol{N}_{0}}=h_{1} \cdot \varphi_{2}$. Then, it is easily seen that $H(p) \in O(m)$ and the equalities (3.3) and (3.4) are satisfied.

Therefore, by Theorem 6 we know that $\boldsymbol{f}_{1}$ can be written as $\boldsymbol{f}_{1}=a \boldsymbol{f}_{0}$, where $a$ denotes the euclidean transformation of $\boldsymbol{R}^{m}$ defined by $\boldsymbol{R}^{m} \ni \boldsymbol{x} \longmapsto$ $H_{0} \cdot \boldsymbol{x}+\boldsymbol{c}_{0} \in \boldsymbol{R}^{m}$. Thus, we obtain the theorem.

\section{The Cayley projective plane $P^{2}(\mathrm{Cay})$}

Let $M=G / K$ be a compact Riemannian symmetric space. Let $\mathfrak{g}$ (resp. $\mathfrak{k}$ ) be the Lie algebra of $G$ (resp. $K$ ). We denote by $\mathfrak{g}=\mathfrak{k}+\mathfrak{m}$ the canonical decomposition of $\mathfrak{g}$ associated with the symmetric pair $(G, K)$. We denote by $($,$) the inner product of \mathfrak{g}$ given by the $(-1)$-multiple of the Killing form of $\mathfrak{g}$. As usual, we can identify $\mathfrak{m}$ with the tangent space $T_{o}(G / K)$ at the origin $o=\{K\}$. We assume that the $G$-invariant Riemannian metric $g$ of $G / K$ satisfies

$$
g_{o}(X, Y)=(X, Y), \quad \forall X, Y \in \mathfrak{m} .
$$

Then, it is well-known that at the origin $o$ the Riemannian curvature tensor $R$ of type $(1,3)$ is given by 


$$
R_{o}(X, Y) Z=-[[X, Y], Z], \quad X, Y, Z \in \mathfrak{m} .
$$

Hereafter, we consider the case of the Cayley projective plane $P^{2}(\boldsymbol{C a y})$. As is well-known, $P^{2}(\boldsymbol{C a y})$ can be represented by $P^{2}(\boldsymbol{C a y})=G / K$, where $G=F_{4}$ and $K=\operatorname{Spin}(9)$. Take a maximal abelian subspace $\mathfrak{a}$ of $\mathfrak{m}$ and fix it in the following discussions. We note that $\operatorname{since} \operatorname{rank}\left(P^{2}(\boldsymbol{C a y})\right)=1$, we have $\operatorname{dim} \mathfrak{a}=1$.

For each element $\lambda \in \mathfrak{a}$ we define two subspaces $\mathfrak{k}(\lambda) \subset \mathfrak{k}$ and $\mathfrak{m}(\lambda) \subset \mathfrak{m}$ by

$$
\begin{aligned}
\mathfrak{k}(\lambda) & =\left\{X \in \mathfrak{k} \mid[H,[H, X]]=-(\lambda, H)^{2} X, \quad \forall H \in \mathfrak{a}\right\}, \\
\mathfrak{m}(\lambda) & =\left\{Y \in \mathfrak{m} \mid[H,[H, Y]]=-(\lambda, H)^{2} Y, \quad \forall H \in \mathfrak{a}\right\} .
\end{aligned}
$$

We call $\lambda$ a restricted root if $\mathfrak{m}(\lambda) \neq 0$. Let $\Sigma$ be the set of all non-zero restricted roots. In the case of $P^{2}(\boldsymbol{C a y})$, there is a restricted root $\mu$ such that $\Sigma=\{ \pm \mu, \pm 2 \mu\}$. We take and fix such a restricted root $\mu$. Then we have $\mathfrak{m}(0)=\mathfrak{a}=\boldsymbol{R} \mu$ and

$$
\begin{aligned}
& \mathfrak{k}=\mathfrak{k}(0)+\mathfrak{k}(\mu)+\mathfrak{k}(2 \mu) \quad \text { (orthogonal direct sum), } \\
& \mathfrak{m}=\mathfrak{m}(0)+\mathfrak{m}(\mu)+\mathfrak{m}(2 \mu) \text { (orthogonal direct sum). }
\end{aligned}
$$

(For details, see [6], [7].) For simplicity, for each integer $i$ we set $\mathfrak{k}_{i}=\mathfrak{k}(|i| \mu)$, $\mathfrak{m}_{i}=\mathfrak{m}(|i| \mu)(|i| \leq 2), \mathfrak{k}_{i}=\mathfrak{m}_{i}=0(|i|>2)$. Then we have

Proposition 7 ([7]) (1) Let $i, j=0,1,2$. Then:

$$
\begin{aligned}
& {\left[\mathfrak{k}_{i}, \mathfrak{k}_{j}\right] \subset \mathfrak{k}_{i+j}+\mathfrak{k}_{i-j},} \\
& {\left[\mathfrak{m}_{i}, \mathfrak{m}_{j}\right] \subset \mathfrak{k}_{i+j}+\mathfrak{k}_{i-j},} \\
& {\left[\mathfrak{k}_{i}, \mathfrak{m}_{j}\right] \subset \mathfrak{m}_{i+j}+\mathfrak{m}_{i-j} .}
\end{aligned}
$$

(2) $\operatorname{dim} \mathfrak{m}=16, \operatorname{dim} \mathfrak{k}_{1}=\operatorname{dim} \mathfrak{m}_{1}=8, \operatorname{dim} \mathfrak{k}_{2}=\operatorname{dim} \mathfrak{m}_{2}=7$.

In what follows, we recall the results obtained in [7], which will be needed in the proof of Theorem 2. Let $V$ be a subspace of $\mathfrak{m}$. $V$ is called pseudo-abelian if it satisfies $[V, V] \subset \mathfrak{k}_{0}$ (or equivalently $[[V, V], \mathfrak{a}]=0$ ). (Precisely, see [6].) As is easily seen, $\mathfrak{m}_{2}$ is a pseudo-abelian subspace of $\mathfrak{m}$, because $\left[\mathfrak{m}_{2}, \mathfrak{m}_{2}\right] \subset \mathfrak{k}_{0}($ see $(4.1))$.

On the contrary, we have

Proposition 8 Let $G / K=P^{2}(\boldsymbol{C a y})$. Then, any pseudo-abelian subspace $V$ of $\mathfrak{m}$ with $\operatorname{dim} V>2$ must be contained in $\mathfrak{m}_{2}$. 
For the proof, see Lemma 6 of [7]. The following proposition summarizes the results of [7] (see Proposition 7, Proposition 10 and Lemma 17 of [7]).

Proposition 9 (1) Let $Y_{0} \in \mathfrak{a}+\mathfrak{m}_{2}$ and $Y_{1} \in \mathfrak{m}_{1}$. Assume that $Y_{0} \neq 0$, $Y_{1} \neq 0$. Then, there are elements $k_{0}, k_{1} \in K$ satisfying

$$
\begin{aligned}
& \operatorname{Ad}\left(k_{0}\right) \mu \in \boldsymbol{R} Y_{0}, \quad \operatorname{Ad}\left(k_{0}\right) \mathfrak{m}_{2}=\left\{Y_{0}^{\prime} \in \mathfrak{a}+\mathfrak{m}_{2} \mid\left(Y_{0}^{\prime}, Y_{0}\right)=0\right\}, \\
& \operatorname{Ad}\left(k_{1}\right) \mu \in \boldsymbol{R} Y_{1}, \quad \operatorname{Ad}\left(k_{1}\right) \mathfrak{m}_{2}=\left\{Y_{1}^{\prime} \in \mathfrak{m}_{1} \mid\left(Y_{1}^{\prime}, Y_{1}\right)=0\right\}
\end{aligned}
$$

(2) Let $Y_{0}, Y_{0}^{\prime} \in \mathfrak{a}+\mathfrak{m}_{2}, Y_{1}, Y_{1}^{\prime} \in \mathfrak{m}_{1}$ and $X_{1} \in \mathfrak{k}_{1}$. Then:

$$
\begin{aligned}
& {\left[Y_{0},\left[Y_{0}, Y_{0}^{\prime}\right]\right]=\left\{\begin{array}{cl}
-4(\mu, \mu)\left(Y_{0}, Y_{0}\right) Y_{0}^{\prime}, & \text { if }\left(Y_{0}, Y_{0}^{\prime}\right)=0, \\
0, & \text { if } Y_{0}^{\prime} \in \boldsymbol{R} Y_{0},
\end{array}\right.} \\
& {\left[Y_{0},\left[Y_{0}, Y_{1}\right]\right]=-(\mu, \mu)\left(Y_{0}, Y_{0}\right) Y_{1},} \\
& {\left[Y_{1},\left[Y_{1}, Y_{0}\right]\right]=-(\mu, \mu)\left(Y_{1}, Y_{1}\right) Y_{0},} \\
& {\left[Y_{1},\left[Y_{1}, Y_{1}^{\prime}\right]\right]=\left\{\begin{array}{cl}
-4(\mu, \mu)\left(Y_{1}, Y_{1}\right) Y_{1}^{\prime}, & \text { if }\left(Y_{1}, Y_{1}^{\prime}\right)=0, \\
0, & \text { if } Y_{1}^{\prime} \in \boldsymbol{R} Y_{1},
\end{array}\right.} \\
& {\left[X_{1},\left[X_{1}, Y_{0}\right]\right]=-(\mu, \mu)\left(X_{1}, X_{1}\right) Y_{0} .}
\end{aligned}
$$

\section{Solutions of the Gauss equation}

In this and the next sections, we prove

Theorem 10 The projective plane $P^{2}(\boldsymbol{C a y})$ is formally rigid in codimension $10\left(=26-\operatorname{dim} P^{2}(\boldsymbol{C a y})\right)$.

If this theorem is established, then Theorem 2 immediately follows from Theorem 5 .

On account of homogeneity of $P^{2}(\boldsymbol{C a y})$, in order to show Theorem 10 we have only to prove that the Gaussian variety $\mathcal{G}_{o}(\boldsymbol{N})$ is EOS at the origin $o$ for any euclidean vector space $\boldsymbol{N}$ with $\operatorname{dim} \boldsymbol{N}=10$.

In what follows we assume that $M=P^{2}(\boldsymbol{C a y})$ and that $\boldsymbol{N}$ is a euclidean vector space with $\operatorname{dim} \boldsymbol{N}=10$. We will prove the following theorem:

Theorem 11 Let $\boldsymbol{\Psi} \in \mathcal{G}_{o}(\boldsymbol{N})$. Then:

(1) There are linearly independent vectors $\mathbf{A}$ and $\mathbf{B} \in \boldsymbol{N}$ satisfying

(1a) $\langle\mathbf{A}, \mathbf{A}\rangle=\langle\mathbf{B}, \mathbf{B}\rangle=4(\mu, \mu)$ and $\langle\mathbf{A}, \mathbf{B}\rangle=2(\mu, \mu)$;

(1b) $\quad \boldsymbol{\Psi}\left(Y_{0}, Y_{0}^{\prime}\right)=\left(Y_{0}, Y_{0}^{\prime}\right) \mathbf{A}, \quad \forall Y_{0}, Y_{0}^{\prime} \in \mathfrak{a}+\mathfrak{m}_{2}$ 
(1c) $\boldsymbol{\Psi}\left(Y_{1}, Y_{1}^{\prime}\right)=\left(Y_{1}, Y_{1}^{\prime}\right) \mathbf{B}, \quad \forall Y_{1}, Y_{1}^{\prime} \in \mathfrak{m}_{1}$;

(1d) $\left\langle\mathbf{A}, \boldsymbol{\Psi}\left(\mu, \mathfrak{m}_{1}\right)\right\rangle=\left\langle\mathbf{B}, \boldsymbol{\Psi}\left(\mu, \mathfrak{m}_{1}\right)\right\rangle=0$.

(2) $\boldsymbol{\Psi}\left(Y_{1}, Y_{2}\right)+\left(1 /(\mu, \mu)^{2}\right) \boldsymbol{\Psi}\left(\mu,\left[\left[\mu, Y_{1}\right], Y_{2}\right]\right)=0, \quad \forall Y_{1} \in \mathfrak{m}_{1}, \forall Y_{2} \in$ $\mathfrak{m}_{2}$.

(3) $\left\langle\Psi\left(\mu, Y_{1}\right), \boldsymbol{\Psi}\left(\mu, Y_{1}^{\prime}\right)\right\rangle=(\mu, \mu)^{2}\left(Y_{1}, Y_{1}^{\prime}\right), \quad \forall Y_{1}, Y_{1}^{\prime} \in \mathfrak{m}_{1}$.

Before proceeding to the proof of Theorem 11 we make a somewhat lengthy preparation. Let $\boldsymbol{N}$ be a euclidean vector space and let $S^{2} \mathfrak{m}^{*} \otimes \boldsymbol{N}$ be the space of $\boldsymbol{N}$-valued symmetric bilinear forms on $\mathfrak{m}$. Let $\boldsymbol{\Psi} \in S^{2} \mathfrak{m}^{*} \otimes$ $\boldsymbol{N}$ and $Y \in \mathfrak{m}$. We define a linear map $\boldsymbol{\Psi}_{Y}$ of $\mathfrak{m}$ to $\boldsymbol{N}$ by

$$
\boldsymbol{\Psi}_{Y}: \mathfrak{m} \ni Y^{\prime} \longmapsto \boldsymbol{\Psi}\left(Y, Y^{\prime}\right) \in \boldsymbol{N}
$$

and denote by $\operatorname{Ker}\left(\boldsymbol{\Psi}_{Y}\right)$ the kernel of $\boldsymbol{\Psi}_{Y}$. We say that an element $Y \in \mathfrak{m}$ is singular (resp. non-singular) with respect to $\boldsymbol{\Psi}$ if $\boldsymbol{\Psi}_{Y}(\mathfrak{m}) \neq \boldsymbol{N}$ (resp. $\left.\boldsymbol{\Psi}_{Y}(\mathfrak{m})=\boldsymbol{N}\right)$. Apparently, $0(\in \mathfrak{m})$ is a singular element for any $\boldsymbol{\Psi} \in$ $S^{2} \mathfrak{m}^{*} \otimes N$.

Proposition 12 Let $\boldsymbol{\Psi} \in \mathcal{G}_{o}(\boldsymbol{N})$. Let $Y \in \mathfrak{m}(Y \neq 0)$ and let $k$ be an element of $K$ satisfying $\operatorname{Ad}(k) \mu \in \boldsymbol{R} Y$. Then:

(1) $\operatorname{Ker}\left(\boldsymbol{\Psi}_{Y}\right) \subset \operatorname{Ad}(k) \mathfrak{m}_{2}$. Consequently, $\operatorname{dim} \operatorname{Ker}\left(\boldsymbol{\Psi}_{Y}\right) \leq 7$.

(2) Assume that $Y$ is non-singular with respect to $\boldsymbol{\Psi}$. Then, it holds that $\operatorname{dim} \operatorname{Ker}\left(\boldsymbol{\Psi}_{Y}\right)=6$ and $\operatorname{Ker}\left(\boldsymbol{\Psi}_{Y}\right) \subsetneq \operatorname{Ad}(k) \mathfrak{m}_{2}$.

(3) Assume that $Y$ is singular with respect to $\boldsymbol{\Psi}$. Then, it holds that $\operatorname{Ker}\left(\boldsymbol{\Psi}_{Y}\right)=\operatorname{Ad}(k) \mathfrak{m}_{2}, \operatorname{dim} \boldsymbol{K e r}\left(\boldsymbol{\Psi}_{Y}\right)=7$ and $\operatorname{dim} \boldsymbol{\Psi}_{Y}(\mathfrak{m})=9$.

Proof. First, note that $\operatorname{dim} \operatorname{Ker}\left(\boldsymbol{\Psi}_{Y}\right) \geq \operatorname{dim} \mathfrak{m}-\operatorname{dim} \boldsymbol{N}=6$. Consequently, it is easy to see that $Y$ is singular (resp. non-singular) with respect to $\boldsymbol{\Psi}$ if and only if $\operatorname{dim} \operatorname{Ker}\left(\boldsymbol{\Psi}_{Y}\right)>6\left(\right.$ resp. $\left.\operatorname{dim} \operatorname{Ker}\left(\boldsymbol{\Psi}_{Y}\right)=6\right)$.

Multiplying $Y$ by a non-zero scalar if necessary, we may assume that $Y=\operatorname{Ad}(k) \mu$. From the Gauss equation (2.1) it follows that

$$
R_{o}\left(\operatorname{Ker}\left(\boldsymbol{\Psi}_{Y}\right), \boldsymbol{K e r}\left(\boldsymbol{\Psi}_{Y}\right)\right) Y=0 .
$$

In our terminology we have

$$
\left[\left[\operatorname{Ker}\left(\boldsymbol{\Psi}_{Y}\right), \operatorname{Ker}\left(\boldsymbol{\Psi}_{Y}\right)\right], Y\right]=0 .
$$

Applying $\operatorname{Ad}\left(k^{-1}\right)$ to the both sides of the above equality, we have

$$
\left[\left[\operatorname{Ad}\left(k^{-1}\right) \operatorname{Ker}\left(\Psi_{Y}\right), \operatorname{Ad}\left(k^{-1}\right) \operatorname{Ker}\left(\Psi_{Y}\right)\right], \mu\right]=0 .
$$


Since $\mathfrak{a}=\boldsymbol{R} \mu$, it follows that $\operatorname{Ad}\left(k^{-1}\right) \operatorname{Ker}\left(\boldsymbol{\Psi}_{Y}\right)$ is a pseudo-abelian subspace of $\mathfrak{m}$. By Proposition 8 and by the fact $\operatorname{dim} \operatorname{Ker}\left(\boldsymbol{\Psi}_{Y}\right) \geq 6$, we have $\operatorname{Ad}\left(k^{-1}\right) \operatorname{Ker}\left(\boldsymbol{\Psi}_{Y}\right) \subset \mathfrak{m}_{2}$ and hence $\operatorname{Ker}\left(\boldsymbol{\Psi}_{Y}\right) \subset \operatorname{Ad}(k) \mathfrak{m}_{2}$, proving (1).

Assume that $Y$ is non-singular with respect to $\boldsymbol{\Psi}$. Then, as we have stated above, we have $\operatorname{dim} \operatorname{Ker}\left(\boldsymbol{\Psi}_{Y}\right)=6$. Since $\operatorname{dim} \mathfrak{m}_{2}=7$ (see Proposition $7(2))$, it follows that $\operatorname{Ker}\left(\boldsymbol{\Psi}_{Y}\right) \subsetneq \operatorname{Ad}(k) \mathfrak{m}_{2}$, proving (2).

Finally, we assume $Y$ is singular with respect to $\boldsymbol{\Psi}$. Then, we have $\operatorname{dim} \operatorname{Ker}\left(\boldsymbol{\Psi}_{Y}\right)>6$. Since $\operatorname{Ker}\left(\boldsymbol{\Psi}_{Y}\right) \subset \operatorname{Ad}(k) \mathfrak{m}_{2}$ and since $\operatorname{dim} \mathfrak{m}_{2}=7$, we have $\operatorname{dim} \operatorname{Ker}\left(\boldsymbol{\Psi}_{Y}\right)=7$ and $\operatorname{Ker}\left(\boldsymbol{\Psi}_{Y}\right)=\operatorname{Ad}(k) \mathfrak{m}_{2}$. This proves (3).

Corollary 13 Let $\boldsymbol{\Psi} \in \mathcal{G}_{o}(\boldsymbol{N})$. Let $Y_{0} \in \mathfrak{a}+\mathfrak{m}_{2}\left(Y_{0} \neq 0\right)$ and $Y_{1} \in$ $\mathfrak{m}_{1}\left(Y_{1} \neq 0\right)$. Then:

(1) $\operatorname{Ker}\left(\boldsymbol{\Psi}_{Y_{0}}\right) \subset\left\{Y_{0}^{\prime} \in \mathfrak{a}+\mathfrak{m}_{2} \mid\left(Y_{0}^{\prime}, Y_{0}\right)=0\right\}$. In particular, if $Y_{0}$ is singular with respect to $\boldsymbol{\Psi}$, then $\operatorname{Ker}\left(\boldsymbol{\Psi}_{Y_{0}}\right)=\left\{Y_{0}^{\prime} \in \mathfrak{a}+\mathfrak{m}_{2} \mid\left(Y_{0}^{\prime}, Y_{0}\right)=0\right\}$.

(2) $\operatorname{Ker}\left(\boldsymbol{\Psi}_{Y_{1}}\right) \subset\left\{Y_{1}^{\prime} \in \mathfrak{m}_{1} \mid\left(Y_{1}^{\prime}, Y_{1}\right)=0\right\}$. In particular, if $Y_{1}$ is singular with respect to $\mathbf{\Psi}$, then $\operatorname{Ker}\left(\mathbf{\Psi}_{Y_{1}}\right)=\left\{Y_{1}^{\prime} \in \mathfrak{m}_{1} \mid\left(Y_{1}^{\prime}, Y_{1}\right)=0\right\}$.

Proof. Let $Y_{0} \in \mathfrak{a}+\mathfrak{m}_{2}\left(Y_{0} \neq 0\right)$. By Proposition 9 (1), we know that there is an element $k_{0} \in K$ satisfying (4.2). Applying Proposition 12 to $Y_{0}$, we easily get $\operatorname{Ker}\left(\boldsymbol{\Psi}_{Y_{0}}\right) \subset\left\{Y_{0}^{\prime} \in \mathfrak{a}+\mathfrak{m}_{2} \mid\left(Y_{0}^{\prime}, Y_{0}\right)=0\right\}$. Assume that $Y_{0}$ is singular with respect to $\boldsymbol{\Psi}$. Then, by the equality $\operatorname{Ker}\left(\boldsymbol{\Psi}_{Y_{0}}\right)=\operatorname{Ad}\left(k_{0}\right) \mathfrak{m}_{2}$, we get (1).

The assertion (2) is similarly dealt with.

Let $\boldsymbol{\Psi} \in S^{2} \mathfrak{m}^{*} \otimes \boldsymbol{N}$. A subspace $U$ of $\mathfrak{m}$ is called singular with respect to $\boldsymbol{\Psi}$ if each element of $U$ is singular with respect to $\boldsymbol{\Psi}$.

Proposition 14 Let $\boldsymbol{\Psi} \in \mathcal{G}_{o}(\boldsymbol{N})$. Let $Y \in \mathfrak{m}(Y \neq 0)$ and let $k \in K$ satisfy $\operatorname{Ad}(k) \mu \in \boldsymbol{R} Y$. Assume that $Y$ is non-singular with respect to $\boldsymbol{\Psi}$. Then:

(1) $\operatorname{Ker}\left(\boldsymbol{\Psi}_{Y}\right)$ is a singular subspace with respect to $\boldsymbol{\Psi}$.

(2) There is an element $Y^{\prime} \in \operatorname{Ad}(k) \mathfrak{m}_{2}$ satisfying $\boldsymbol{\Psi}\left(Y, Y^{\prime}\right) \neq 0$ and

$$
\boldsymbol{N}=\boldsymbol{R} \boldsymbol{\Psi}\left(Y, Y^{\prime}\right)+\boldsymbol{\Psi}_{Y^{\prime \prime}}(\mathfrak{m}) \quad \text { (orthogonal direct sum), }
$$

where $Y^{\prime \prime}$ is an arbitrary non-zero element of $\operatorname{Ker}\left(\boldsymbol{\Psi}_{Y}\right)$.

Proof. Since $Y$ is non-singular with respect to $\boldsymbol{\Psi}$, we have $\operatorname{Ker}\left(\boldsymbol{\Psi}_{Y}\right) \subsetneq$ $\operatorname{Ad}(k) \mathfrak{m}_{2}$ (see Proposition 12). Take a non-zero element $Y^{\prime} \in \operatorname{Ad}(k) \mathfrak{m}_{2}$ such that $\left(Y^{\prime}, \boldsymbol{K e r}\left(\boldsymbol{\Psi}_{Y}\right)\right)=0$. Then, since $Y^{\prime} \notin \operatorname{Ker}\left(\boldsymbol{\Psi}_{Y}\right)$, we have $\boldsymbol{\Psi}\left(Y, Y^{\prime}\right) \neq$ 0 
Let $Y^{\prime \prime} \in \operatorname{Ker}\left(\boldsymbol{\Psi}_{Y}\right)\left(Y^{\prime \prime} \neq 0\right)$. Then, by the Gauss equation (2.1) we have

$$
\begin{aligned}
& \left(\left[\left[Y^{\prime}, Y^{\prime \prime}\right], Y\right], W\right) \\
& =\left\langle\boldsymbol{\Psi}\left(Y^{\prime}, Y\right), \boldsymbol{\Psi}\left(Y^{\prime \prime}, W\right)\right\rangle-\left\langle\boldsymbol{\Psi}\left(Y^{\prime}, W\right), \mathbf{\Psi}\left(Y^{\prime \prime}, Y\right)\right\rangle,
\end{aligned}
$$

where $W$ is an arbitrary element of $\mathfrak{m}$. Note that the left hand side of (5.2) vanishes, because

$$
\begin{array}{r}
{\left[\left[Y^{\prime}, Y^{\prime \prime}\right], Y\right] \in\left[\left[\operatorname{Ad}(k) \mathfrak{m}_{2}, \operatorname{Ad}(k) \mathfrak{m}_{2}\right], \operatorname{Ad}(k) \mu\right]} \\
=\operatorname{Ad}(k)\left[\left[\mathfrak{m}_{2}, \mathfrak{m}_{2}\right], \mu\right]=0 .
\end{array}
$$

We also note that $\boldsymbol{\Psi}\left(Y^{\prime \prime}, Y\right)=0$, because $Y^{\prime \prime} \in \operatorname{Ker}\left(\boldsymbol{\Psi}_{Y}\right)$. Consequently, we have $\left\langle\boldsymbol{\Psi}\left(Y^{\prime}, Y\right), \boldsymbol{\Psi}\left(Y^{\prime \prime}, W\right)\right\rangle=0$. This implies that each element of $\boldsymbol{\Psi}_{Y^{\prime \prime}}(\mathfrak{m})$ is orthogonal to $\boldsymbol{\Psi}\left(Y^{\prime}, Y\right)$. Therefore, $\boldsymbol{\Psi}_{Y^{\prime \prime}}(\mathfrak{m}) \neq \boldsymbol{N}$, implying that $Y^{\prime \prime}$ is singular with respect to $\boldsymbol{\Psi}$. Hence, by Proposition 12 (3) we have $\operatorname{dim} \boldsymbol{\Psi}_{Y^{\prime \prime}}(\mathfrak{m})=9$, which proves (5.1).

The following lemma assures that for each $\boldsymbol{\Psi} \in \mathcal{G}_{o}(\boldsymbol{N})$ there are many high dimensional singular subspaces with respect to $\boldsymbol{\Psi}$.

Lemma 15 Let $\boldsymbol{\Psi} \in \mathcal{G}_{o}(\boldsymbol{N})$. Then, there are singular subspaces $U$ and $V$ with respect to $\Psi$ satisfying $U \subset \mathfrak{a}+\mathfrak{m}_{2}, V \subset \mathfrak{m}_{1}, \operatorname{dim} U \geq 6$ and $\operatorname{dim} V \geq 6$.

Proof. If $\mathfrak{a}+\mathfrak{m}_{2}$ contains no non-singular element with respect to $\boldsymbol{\Psi}$, then we can take $U=\mathfrak{a}+\mathfrak{m}_{2}$. (Note that $\operatorname{dim}\left(\mathfrak{a}+\mathfrak{m}_{2}\right)=8$.) On the contrary, if $\mathfrak{a}+\mathfrak{m}_{2}$ contains a non-singular element $Y_{0}$, then we set $U=\operatorname{Ker}\left(\boldsymbol{\Psi}_{Y_{0}}\right)$. Then, we know that $U \subset \mathfrak{a}+\mathfrak{m}_{2}, \operatorname{dim} U=6$ (see Proposition 12 (2) and Corollary $13(1)$ ) and that $U$ is a singular subspace with respect to $\boldsymbol{\Psi}$ (see Proposition 14 (1)). Similarly, we can select a singular subspace $V \subset \mathfrak{m}_{1}$ with $\operatorname{dim} V \geq 6$.

Proposition 16 Let $\boldsymbol{\Psi} \in \mathcal{G}_{o}(\boldsymbol{N})$. Let $U$ and $V$ be arbitrary singular subspaces with respect to $\boldsymbol{\Psi}$ satisfying $U \subset \mathfrak{a}+\mathfrak{m}_{2}, V \subset \mathfrak{m}_{1}$, $\operatorname{dim} U \geq 6$ and $\operatorname{dim} V \geq 6$. Then there are two vectors $\mathbf{A}$ and $\mathbf{B} \in \boldsymbol{N}$ satisfying:

(1) $\langle\mathbf{A}, \mathbf{A}\rangle=\langle\mathbf{B}, \mathbf{B}\rangle=4(\mu, \mu)$

(2) $\boldsymbol{\Psi}\left(\xi, Y_{0}\right)=\left(\xi, Y_{0}\right) \mathbf{A}, \quad \forall \xi \in U, \forall Y_{0} \in \mathfrak{a}+\mathfrak{m}_{2}$;

(3) $\boldsymbol{\Psi}\left(\eta, Y_{1}\right)=\left(\eta, Y_{1}\right) \mathbf{B}, \quad \forall \eta \in V, \forall Y_{1} \in \mathfrak{m}_{1}$;

(4) $\left\langle\mathbf{A}, \Psi_{Y_{0}}\left(\mathfrak{m}_{1}\right)\right\rangle=\left\langle\mathbf{B}, \Psi_{Y_{0}}\left(\mathfrak{m}_{1}\right)\right\rangle=0, \quad \forall Y_{0} \in \mathfrak{a}+\mathfrak{m}_{2}$. 
Proof. Let $\xi \in U(\xi \neq 0)$. Since $\xi$ is singular with respect to $\boldsymbol{\Psi}, \operatorname{Ker}\left(\mathbf{\Psi}_{\xi}\right)$ coincides with the orthogonal complement of $\boldsymbol{R} \xi$ in $\mathfrak{a}+\mathfrak{m}_{2}$ (see Corollary $13(1))$. Hence, the equality $\boldsymbol{\Psi}\left(\xi, Y_{0}\right)=0$ holds for each $Y_{0} \in \mathfrak{a}+$ $\mathfrak{m}_{2}$ satisfying $\left(\xi, Y_{0}\right)=0$. In particular, we have

$$
\boldsymbol{\Psi}\left(\xi, \xi^{\prime}\right)=0, \quad \forall \xi, \xi^{\prime} \in U \text { with }\left(\xi, \xi^{\prime}\right)=0 .
$$

Then, applying the same argument as in the proof of Proposition 9 of [7], we can prove that there is a vector $\mathbf{A} \in \boldsymbol{N}$ satisfying

$$
\boldsymbol{\Psi}\left(\xi, \xi^{\prime}\right)=\left(\xi, \xi^{\prime}\right) \mathbf{A}, \quad \forall \xi, \xi^{\prime} \in U .
$$

Let $Y_{0} \in \mathfrak{a}+\mathfrak{m}_{2}$ satisfy $\left(Y_{0}, U\right)=0$. Then, since $\left(\xi, Y_{0}\right)=0$, we have $\boldsymbol{\Psi}\left(\xi, Y_{0}\right)=0$ and $\left(\xi, Y_{0}\right) \mathbf{A}=0$. This, together with (5.3), proves (2). The assertion (3) can be proved in the same way.

We now prove (1). Let $\xi, \xi^{\prime} \in U$ satisfy $\left(\xi, \xi^{\prime}\right)=0$ and $(\xi, \xi)=$ $\left(\xi^{\prime}, \xi^{\prime}\right)=1$. Put $X=Z=\xi$ and $Y=W=\xi^{\prime}$ into the Gauss equation (2.1). Then, we have

$$
\left(\left[\left[\xi, \xi^{\prime}\right], \xi\right], \xi^{\prime}\right)=\left\langle\boldsymbol{\Psi}(\xi, \xi), \mathbf{\Psi}\left(\xi^{\prime}, \xi^{\prime}\right)\right\rangle-\left\langle\boldsymbol{\Psi}\left(\xi, \xi^{\prime}\right), \boldsymbol{\Psi}\left(\xi^{\prime}, \xi\right)\right\rangle .
$$

Since $\left[\left[\xi, \xi^{\prime}\right], \xi\right]=4(\mu, \mu) \xi^{\prime}$ (see $\left.(4.4)\right), \boldsymbol{\Psi}(\xi, \xi)=\boldsymbol{\Psi}\left(\xi^{\prime}, \xi^{\prime}\right)=\mathbf{A}$ and $\boldsymbol{\Psi}\left(\xi, \xi^{\prime}\right)=0$, we have $\langle\mathbf{A}, \mathbf{A}\rangle=4(\mu, \mu)$. Similarly, by (4.7) we can prove $\langle\mathbf{B}, \mathbf{B}\rangle=4(\mu, \mu)$, proving (1).

Finally, we prove (4). Let $Y_{1} \in \mathfrak{m}_{1}$ and $Y_{0} \in \mathfrak{a}+\mathfrak{m}_{2}$. Take an element $\xi \in$ $U$ satisfying $\left(\xi, Y_{0}\right)=0$ and $(\xi, \xi)=1$. Such $\xi$ can exist, because $\operatorname{dim} U \geq$ 6. Put $X=Z=\xi, Y=Y_{0}$ and $W=Y_{1}$ into the Gauss equation (2.1). Then we have

$$
\left(\left[\left[\xi, Y_{0}\right], \xi\right], Y_{1}\right)=\left\langle\Psi(\xi, \xi), \mathbf{\Psi}\left(Y_{0}, Y_{1}\right)\right\rangle-\left\langle\Psi\left(\xi, Y_{1}\right), \mathbf{\Psi}\left(Y_{0}, \xi\right)\right\rangle .
$$

Since $\left(\xi, Y_{0}\right)=0$, we have $\boldsymbol{\Psi}\left(\xi, Y_{0}\right)=0$ and $\left[\left[\xi, Y_{0}\right], \xi\right]=4(\mu, \mu) Y_{0}$ (see (4.4)). Moreover, since $\mathbf{\Psi}(\xi, \xi)=\mathbf{A}$ and $\left(Y_{0}, Y_{1}\right)=0$, we have

$$
\begin{aligned}
\left\langle\mathbf{A}, \boldsymbol{\Psi}_{Y_{0}}\left(Y_{1}\right)\right\rangle & =\left\langle\boldsymbol{\Psi}(\xi, \xi), \mathbf{\Psi}\left(Y_{0}, Y_{1}\right)\right\rangle \\
& =\left\langle\boldsymbol{\Psi}\left(\xi, Y_{1}\right), \boldsymbol{\Psi}\left(Y_{0}, \xi\right)\right\rangle+4(\mu, \mu)\left(Y_{0}, Y_{1}\right) \\
& =0 .
\end{aligned}
$$

Since $Y_{1}$ is an arbitrary element of $\mathfrak{m}_{1}$, we have $\left\langle\mathbf{A}, \boldsymbol{\Psi}_{Y_{0}}\left(\mathfrak{m}_{1}\right)\right\rangle=0$. In a similar way, the equality $\left\langle\mathbf{B}, \mathbf{\Psi}_{Y_{0}}\left(\mathfrak{m}_{1}\right)\right\rangle=0$ can be proved. 
Remark 4 As seen in the proof of Lemma 15, singular subspaces $U$ and $V$ may not be uniquely determined. However, it is noted that the vectors $\mathbf{A}$ and $\mathbf{B}$ in Proposition 16 do not depend on the choice of $U$ and $V$. In fact, let $U^{\prime}$ and $V^{\prime}$ be different singular subspaces with respect to $\boldsymbol{\Psi}$ satisfying $U^{\prime} \subset \mathfrak{a}+\mathfrak{m}_{2}$ and $V^{\prime} \subset \mathfrak{m}_{1}$ with $\operatorname{dim} U^{\prime} \geq 6, \operatorname{dim} V^{\prime} \geq 6$. Let $\mathbf{A}^{\prime}$ and $\mathbf{B}^{\prime}$ be vectors of $\boldsymbol{N}$ satisfying (1) $\sim(4)$ of Proposition 16 . Then, since $\operatorname{dim}(\mathfrak{a}+$ $\left.\mathfrak{m}_{2}\right)=\operatorname{dim} \mathfrak{m}_{1}=8$, we have $U \cap U^{\prime} \neq 0, V \cap V^{\prime} \neq 0$. Take $\xi \in U \cap U^{\prime}$ and $\eta \in V \cap V^{\prime}$ such that $(\xi, \xi)=(\eta, \eta)=1$. Then we have $\mathbf{A}=\boldsymbol{\Psi}(\xi, \xi)=\mathbf{A}^{\prime}$ and $\mathbf{B}=\mathbf{\Psi}(\eta, \eta)=\mathbf{B}^{\prime}$, showing our assertion.

In the following discussions, we fix an element $\boldsymbol{\Psi} \in \mathcal{G}_{o}(\boldsymbol{N})$, singular subspaces $U, V$ and vectors $\mathbf{A}, \mathbf{B}$ stated in Proposition 16 and prove several lemmas which are indispensable to the proof of Theorem 11.

Lemma 17 Let $\xi \in U, \eta \in V, Y_{0} \in \mathfrak{a}+\mathfrak{m}_{2}$ and $Y_{1} \in \mathfrak{m}_{1}$. Set $C=$ $\langle\mathbf{A}, \mathbf{B}\rangle-(\mu, \mu)$. Then $C>0$ and:

(1) $\left\langle\boldsymbol{\Psi}_{Y_{0}}(\eta), \boldsymbol{\Psi}_{Y_{0}}\left(Y_{1}\right)\right\rangle=\left\{\left\langle\boldsymbol{\Psi}\left(Y_{0}, Y_{0}\right), \mathbf{B}\right\rangle-(\mu, \mu)\left(Y_{0}, Y_{0}\right)\right\}\left(\eta, Y_{1}\right)$;

(2) $\left\langle\boldsymbol{\Psi}_{\xi}(\eta), \boldsymbol{\Psi}_{\xi}\left(Y_{1}\right)\right\rangle=C(\xi, \xi)\left(\eta, Y_{1}\right)$.

Proof. Putting $X=Z=Y_{0}, Y=Y_{1}$ and $W=\eta$ into (2.1), we have

$$
\left(\left[\left[Y_{0}, Y_{1}\right], Y_{0}\right], \eta\right)=\left\langle\boldsymbol{\Psi}\left(Y_{0}, Y_{0}\right), \boldsymbol{\Psi}\left(Y_{1}, \eta\right)\right\rangle-\left\langle\boldsymbol{\Psi}\left(Y_{0}, \eta\right), \mathbf{\Psi}\left(Y_{1}, Y_{0}\right)\right\rangle .
$$

Since $\left[\left[Y_{0}, Y_{1}\right], Y_{0}\right]=(\mu, \mu)\left(Y_{0}, Y_{0}\right) Y_{1}$ (see $\left.(4.5)\right)$ and $\mathbf{\Psi}\left(Y_{1}, \eta\right)=\left(Y_{1}, \eta\right) \mathbf{B}$, we easily get (1). Putting $Y_{0}=\xi \in U$ into (1), we easily have (2). If we set $Y_{1}=\eta \in V$ in $(2)$, we have $\left\langle\boldsymbol{\Psi}_{\xi}(\eta), \mathbf{\Psi}_{\xi}(\eta)\right\rangle=C(\xi, \xi)(\eta, \eta)$. Since $\operatorname{Ker}\left(\boldsymbol{\Psi}_{\xi}\right) \cap \mathfrak{m}_{1}=0$ (see Corollary $13(1)$ ), we have $\boldsymbol{\Psi}_{\xi}(\eta) \neq 0$ if $\eta \neq 0$. Consequently, we have $C>0$.

Let $Y_{0} \in \mathfrak{a}+\mathfrak{m}_{2}$. Let $\xi^{0}$ be a non-zero element of $U$ satisfying $\left(\xi^{0}, Y_{0}\right)=$ 0 . (Such $\xi^{0}$ exists, because $\operatorname{dim} U \geq 6$.) We define a linear mapping $\Theta_{Y_{0}, \xi^{0}}: V \longrightarrow \boldsymbol{N}$ by

$$
\boldsymbol{\Theta}_{Y_{0}, \xi^{0}}(\eta)=\mathbf{\Psi}_{Y_{0}}(\eta)+\frac{1}{C\left(\xi^{0}, \xi^{0}\right)} \boldsymbol{\Psi}_{\xi^{0}}\left(\left[\left[\xi^{0}, \eta\right], Y_{0}\right]\right), \quad \eta \in V .
$$

Then we have

Lemma $18\left\langle\mathbf{A}, \boldsymbol{\Theta}_{Y_{0}, \xi^{0}}(V)\right\rangle=\left\langle\Psi_{\xi^{0}}(V), \Theta_{Y_{0}, \xi^{0}}(V)\right\rangle=0$.

Proof. We first note that $\left[\left[\xi^{0}, \eta\right], Y_{0}\right] \in \mathfrak{m}_{1}$ for $\eta \in V$ and note that $\boldsymbol{\Theta}_{Y_{0}, \xi^{0}}(V) \subset \boldsymbol{\Psi}_{Y_{0}}\left(\mathfrak{m}_{1}\right)+\boldsymbol{\Psi}_{\xi^{0}}\left(\mathfrak{m}_{1}\right)$. By Proposition 16 (4), we have 
$\left\langle\mathbf{A}, \boldsymbol{\Psi}_{Y_{0}}\left(\mathfrak{m}_{1}\right)\right\rangle=\left\langle\mathbf{A}, \boldsymbol{\Psi}_{\xi^{0}}\left(\mathfrak{m}_{1}\right)\right\rangle=0$ and hence $\left\langle\mathbf{A}, \boldsymbol{\Theta}_{Y_{0}, \xi^{0}}(V)\right\rangle=0$.

Let $\eta, \eta^{\prime} \in V$. Then by putting $X=Y_{0}, Y=\eta^{\prime}, Z=\eta$ and $W=\xi^{0}$ into the Gauss equation (2.1), we have

$$
\begin{aligned}
\left(\left[\left[Y_{0}, \eta^{\prime}\right], \eta\right], \xi^{0}\right) & =\left\langle\mathbf{\Psi}\left(Y_{0}, \eta\right), \mathbf{\Psi}\left(\eta^{\prime}, \xi^{0}\right)\right\rangle-\left\langle\mathbf{\Psi}\left(Y_{0}, \xi^{0}\right), \mathbf{\Psi}\left(\eta^{\prime}, \eta\right)\right\rangle \\
& =\left\langle\mathbf{\Psi}_{Y_{0}}(\eta), \mathbf{\Psi}_{\xi^{0}}\left(\eta^{\prime}\right)\right\rangle-\langle\mathbf{A}, \mathbf{B}\rangle\left(Y_{0}, \xi^{0}\right)\left(\eta^{\prime}, \eta\right) .
\end{aligned}
$$

Since $\left(Y_{0}, \xi^{0}\right)=0$, we have

$$
\left\langle\boldsymbol{\Psi}_{Y_{0}}(\eta), \boldsymbol{\Psi}_{\xi^{0}}\left(\eta^{\prime}\right)\right\rangle=\left(\left[\left[Y_{0}, \eta^{\prime}\right], \eta\right], \xi^{0}\right) .
$$

On the other hand, we have

$$
\left\langle\Psi_{\xi^{0}}\left(\left[\left[\xi^{0}, \eta\right], Y_{0}\right]\right), \Psi_{\xi^{0}}\left(\eta^{\prime}\right)\right\rangle=C\left(\xi^{0}, \xi^{0}\right)\left(\left[\left[\xi^{0}, \eta\right], Y_{0}\right], \eta^{\prime}\right)
$$

(see Lemma 17 (2)). Therefore,

$$
\begin{aligned}
\left\langle\boldsymbol{\Theta}_{Y_{0},}, \xi^{0}\right. & \left.(\eta), \boldsymbol{\Psi}_{\xi^{0}}\left(\eta^{\prime}\right)\right\rangle \\
& =\left\langle\boldsymbol{\Psi}_{Y_{0}}(\eta)+\frac{1}{C\left(\xi^{0}, \xi^{0}\right)} \boldsymbol{\Psi}_{\xi^{0}}\left(\left[\left[\xi^{0}, \eta\right], Y_{0}\right]\right), \boldsymbol{\Psi}_{\xi^{0}}\left(\eta^{\prime}\right)\right\rangle \\
& =\left(\left[\left[Y_{0}, \eta^{\prime}\right], \eta\right], \xi^{0}\right)+\left(\left[\left[\xi^{0}, \eta\right], Y_{0}\right], \eta^{\prime}\right) \\
& =-\left(\left[Y_{0}, \eta^{\prime}\right],\left[\xi^{0}, \eta\right]\right)+\left(\left[\xi^{0}, \eta\right],\left[Y_{0}, \eta^{\prime}\right]\right) \\
& =0 .
\end{aligned}
$$

This completes the proof.

We can further show

Lemma 19 Let $\eta \in V$. Assume that $\left[\left[\xi^{0}, \eta\right], Y_{0}\right] \in V$. Then:

$$
\begin{aligned}
& \left|\boldsymbol{\Theta}_{Y_{0}, \xi^{0}}(\eta)\right|^{2} \\
& \quad=\left[\left\langle\Psi\left(Y_{0}, Y_{0}\right), \mathbf{B}\right\rangle-(\mu, \mu)\left(Y_{0}, Y_{0}\right)\left\{1+\frac{(\mu, \mu)}{C}\right\}\right](\eta, \eta) .
\end{aligned}
$$

Proof. Set $\eta^{\prime}=\left[\left[\xi^{0}, \eta\right], Y_{0}\right]$. By Lemma 18, Lemma 17 and the equality (5.4) we have

$$
\begin{aligned}
& \left\langle\boldsymbol{\Theta}_{Y_{0}, \xi^{0}}(\eta), \boldsymbol{\Theta}_{Y_{0}, \xi^{0}}(\eta)\right\rangle \\
& \quad=\left\langle\boldsymbol{\Psi}_{Y_{0}}(\eta)+\frac{1}{C\left(\xi^{0}, \xi^{0}\right)} \boldsymbol{\Psi}_{\xi^{0}}\left(\eta^{\prime}\right), \boldsymbol{\Theta}_{Y_{0}, \xi^{0}}(\eta)\right\rangle \\
& =\left\langle\boldsymbol{\Psi}_{Y_{0}}(\eta), \boldsymbol{\Theta}_{Y_{0}, \xi^{0}}(\eta)\right\rangle
\end{aligned}
$$




$$
\begin{aligned}
=\left\langle\boldsymbol{\Psi}_{Y_{0}}(\eta), \boldsymbol{\Psi}_{Y_{0}}(\eta)\right\rangle+\frac{1}{C\left(\xi^{0}, \xi^{0}\right)}\left\langle\boldsymbol{\Psi}_{Y_{0}}(\eta), \boldsymbol{\Psi}_{\xi^{0}}\left(\eta^{\prime}\right)\right\rangle \\
=\left\{\left\langle\boldsymbol{\Psi}\left(Y_{0}, Y_{0}\right), \mathbf{B}\right\rangle-(\mu, \mu)\left(Y_{0}, Y_{0}\right)\right\}(\eta, \eta) \\
+\frac{1}{C\left(\xi^{0}, \xi^{0}\right)}\left(\left[\left[Y_{0}, \eta^{\prime}\right], \eta\right], \xi^{0}\right) .
\end{aligned}
$$

Since $\left[\xi^{0}, \eta\right] \in \mathfrak{k}_{1}$, by (4.8) and (4.5) we have

$$
\begin{aligned}
\left(\left[\left[Y_{0}, \eta^{\prime}\right], \eta\right], \xi^{0}\right) & =-\left(\left[Y_{0}, \eta^{\prime}\right],\left[\xi^{0}, \eta\right]\right) \\
& =\left(Y_{0},\left[\left[\xi^{0}, \eta\right], \eta^{\prime}\right]\right) \\
& =\left(Y_{0},\left[\left[\xi^{0}, \eta\right],\left[\left[\xi^{0}, \eta\right], Y_{0}\right]\right]\right) \\
& =-(\mu, \mu)\left(\left[\xi^{0}, \eta\right],\left[\xi^{0}, \eta\right]\right)\left(Y_{0}, Y_{0}\right) \\
& =(\mu, \mu)\left(\left[\xi^{0},\left[\xi^{0}, \eta\right]\right], \eta\right)\left(Y_{0}, Y_{0}\right) \\
& =-(\mu, \mu)^{2}\left(\xi^{0}, \xi^{0}\right)(\eta, \eta)\left(Y_{0}, Y_{0}\right) .
\end{aligned}
$$

Therefore, we obtain (5.5).

Lemma 20 Let $Y_{0} \in \mathfrak{a}+\mathfrak{m}_{2}$. Then:

(1) $\left\langle\boldsymbol{\Psi}\left(Y_{0}, Y_{0}\right), \mathbf{B}\right\rangle=(\mu, \mu)\left(Y_{0}, Y_{0}\right)\{1+(\mu, \mu) / C\}$.

(2) Let $\xi^{0}$ be a non-zero element of $U$ satisfying $\left(Y_{0}, \xi^{0}\right)=0$. Then, $\Theta_{Y_{0}, \xi^{0}}(\eta)=0$, i.e., the equality

$$
\boldsymbol{\Psi}\left(Y_{0}, \eta\right)+\frac{1}{C\left(\xi^{0}, \xi^{0}\right)} \boldsymbol{\Psi}\left(\xi^{0},\left[\left[\xi^{0}, \eta\right], Y_{0}\right]\right)=0
$$

holds for each $\eta \in V$ satisfying $\left[\left[\xi^{0}, \eta\right], Y_{0}\right] \in V$.

Proof. We first show that there is a non-zero element $\eta^{0} \in V$ satisfying $\boldsymbol{\Theta}_{Y_{0}, \xi^{0}}\left(\eta^{0}\right)=0$ and $\left[\left[\xi^{0}, \eta^{0}\right], Y_{0}\right] \in V$. Let $\boldsymbol{D}$ be the orthogonal complement of $\boldsymbol{R} \mathbf{A}+\mathbf{\Psi}_{\xi^{0}}(V)$ in $\boldsymbol{N}$ and let $V^{\prime}$ be the orthogonal complement of $V$ in $\mathfrak{m}_{1}$. By Lemma 18, we easily have $\boldsymbol{\Theta}_{Y_{0}, \xi^{0}}(V) \subset \boldsymbol{D}$. Therefore, to obtain $\eta^{0}$ satisfying the above condition, it suffices to find a non-zero solution $\eta=$ $\eta^{0} \in V$ of the system of linear homogeneous equations

$$
\left\langle\boldsymbol{\Theta}_{Y_{0}, \xi^{0}}(\eta), \boldsymbol{D}\right\rangle=\left(\left[\left[\xi^{0}, \eta\right], Y_{0}\right], V^{\prime}\right)=0 .
$$

Since $\operatorname{Ker}\left(\boldsymbol{\Psi}_{\xi^{0}}\right) \cap \mathfrak{m}_{1}=0$ (see Corollary13 (1)) and $\left\langle\mathbf{A}, \mathbf{\Psi}_{\xi^{0}}\left(\mathfrak{m}_{1}\right)\right\rangle=0$ (see Proposition 16 (4)), we have $\operatorname{dim}\left(\boldsymbol{R A}+\boldsymbol{\Psi}_{\xi^{0}}(V)\right)=1+\operatorname{dim} V \geq 7$. (Recall that we are assuming $V \subset \mathfrak{m}_{1}$ and $\operatorname{dim} V \geq 6$.) Hence, we have $\operatorname{dim} \boldsymbol{D} \leq$ $\operatorname{dim} \boldsymbol{N}-7=3$. Moreover, we have $\operatorname{dim} V^{\prime}=8-\operatorname{dim} V \leq 2$. Consequently, 
the rank of the system (5.7) is less than or equal to 5 . Therefore, we can find a non-zero solution $\eta^{0} \in V$ of (5.7). Putting $\eta=\eta^{0}$ into (5.5), we obtain the equality (1). Further, putting (1) into (5.5), we have $\boldsymbol{\Theta}_{Y_{0}, \xi^{0}}(\eta)=0$ for any $\eta \in V$ satisfying $\left[\left[\xi^{0}, \eta\right], Y_{0}\right] \in V$.

Lemma 21 The vectors $\mathbf{A}$ and $\mathbf{B}$ are linearly independent and $\langle\mathbf{A}, \mathbf{B}\rangle=$ $2(\mu, \mu), C=(\mu, \mu)$.

Proof. Let $\xi \in U$ with $(\xi, \xi)=1$. Since $\boldsymbol{\Psi}(\xi, \xi)=\mathbf{A}$ (see (5.3)), by putting $Y_{0}=\xi$ into the equality in Lemma 20 (1), we easily have $\langle\mathbf{A}, \mathbf{B}\rangle=$ $(\mu, \mu)\{1+(\mu, \mu) / C\}$. Since $C=\langle\mathbf{A}, \mathbf{B}\rangle-(\mu, \mu)$, it immediately follows that $C^{2}=(\mu, \mu)^{2}$. Since $C>0$, we get $C=(\mu, \mu)$ and hence $\langle\mathbf{A}, \mathbf{B}\rangle=$ $2(\mu, \mu)$. This, together with Proposition $16(1)$, proves that $\mathbf{A}$ and $\mathbf{B}$ are linearly independent.

These being prepared, we show Theorem 11.

Proof of Theorem 11. First we show that $\mu$ is singular with respect to any element $\boldsymbol{\Psi} \in \mathcal{G}_{o}(\boldsymbol{N})$. Suppose that there is an element $\boldsymbol{\Psi}_{0} \in \mathcal{G}_{o}(\boldsymbol{N})$ such that $\mu$ is non-singular with respect to $\boldsymbol{\Psi}_{0}$. Then, $\operatorname{Ker}\left(\left(\boldsymbol{\Psi}_{0}\right)_{\mu}\right)$ is a singular subspace with respect to $\boldsymbol{\Psi}_{0}$ and it satisfies $\operatorname{dim} \operatorname{Ker}\left(\left(\boldsymbol{\Psi}_{0}\right)_{\mu}\right)=6$ and $\operatorname{Ker}\left(\left(\boldsymbol{\Psi}_{0}\right)_{\mu}\right) \subset \mathfrak{m}_{2}$ (see Proposition 12 and Proposition 14).

Now, set $\boldsymbol{\Psi}=\boldsymbol{\Psi}_{0}$ and $U=\operatorname{Ker}\left(\left(\boldsymbol{\Psi}_{0}\right)_{\mu}\right)$ in Proposition 16. Let $\mathbf{A}$, B be the vectors of $\boldsymbol{N}$ satisfying (1)-(4) of Proposition 16. Let $\xi \in$ $U=\operatorname{Ker}\left(\left(\boldsymbol{\Psi}_{0}\right)_{\mu}\right)$ with $\xi \neq 0$. First, we show $\mathbf{B} \in\left(\boldsymbol{\Psi}_{0}\right)_{\xi}(\mathfrak{m})$. In fact, there is a non-zero element $Y_{2}^{0} \in \mathfrak{m}_{2}$ satisfying $\boldsymbol{\Psi}_{0}\left(\mu, Y_{2}^{0}\right) \neq 0$ and $\boldsymbol{N}=$ $\boldsymbol{R} \boldsymbol{\Psi}_{0}\left(\mu, Y_{2}^{0}\right)+\left(\boldsymbol{\Psi}_{0}\right)_{\xi}(\mathfrak{m})$ (orthogonal direct sum) (see Proposition 14). By Lemma 20 (1) and by the relation

$$
\boldsymbol{\Psi}_{0}\left(\mu, Y_{2}^{0}\right)=\frac{1}{2}\left(\boldsymbol{\Psi}_{0}\left(\mu+Y_{2}^{0}, \mu+Y_{2}^{0}\right)-\boldsymbol{\Psi}_{0}(\mu, \mu)-\boldsymbol{\Psi}_{0}\left(Y_{2}^{0}, Y_{2}^{0}\right)\right),
$$

we easily have $\left\langle\boldsymbol{\Psi}_{0}\left(\mu, Y_{2}^{0}\right), \mathbf{B}\right\rangle=0$, which proves $\mathbf{B} \in\left(\mathbf{\Psi}_{0}\right)_{\xi}(\mathfrak{m})$. Since $\left(\boldsymbol{\Psi}_{0}\right)_{\xi}(\mathfrak{m})=\boldsymbol{R} \mathbf{A}+\left(\boldsymbol{\Psi}_{0}\right)_{\xi}\left(\mathfrak{m}_{1}\right)$ (orthogonal direct sum) and $\left\langle\mathbf{B},\left(\boldsymbol{\Psi}_{0}\right)_{\xi}\left(\mathfrak{m}_{1}\right)\right\rangle=$ 0 (see Proposition 16 (2), (4)), we have $\mathbf{B} \in \boldsymbol{R A}$. This contradicts Lemma 21. Accordingly, we can conclude that $\mu$ is singular with respect to any element $\boldsymbol{\Psi} \in \mathcal{G}_{o}(\boldsymbol{N})$.

Now we show that any element of $\mathfrak{m}$ is singular with respect to any $\boldsymbol{\Psi} \in \mathcal{G}_{o}(\boldsymbol{N})$. Let $Y$ be a non-zero element of $\mathfrak{m}$. Take an element $k \in K$ such that $\operatorname{Ad}(k) \mu \in \boldsymbol{R} Y$ and define $\boldsymbol{\Psi}^{\prime} \in S^{2} \mathfrak{m}^{*} \otimes \boldsymbol{N}$ by 


$$
\Psi^{\prime}\left(Y^{\prime}, Y^{\prime \prime}\right)=\Psi\left(\operatorname{Ad}(k) Y^{\prime}, \operatorname{Ad}(k) Y^{\prime \prime}\right), \quad Y^{\prime}, Y^{\prime \prime} \in \mathfrak{m} .
$$

Then, it is easily seen that $\boldsymbol{\Psi}^{\prime} \in \mathcal{G}_{o}(\boldsymbol{N})$. Applying the arguments developed above, we know that $\mu$ is also singular with respect to $\boldsymbol{\Psi}^{\prime}$. Note that $\boldsymbol{\Psi}_{\mu}^{\prime}(\mathfrak{m})=\boldsymbol{\Psi}_{\operatorname{Ad}(k) \mu}(\operatorname{Ad}(k) \mathfrak{m})=\boldsymbol{\Psi}_{Y}(\mathfrak{m})$. Then, since $\boldsymbol{\Psi}_{\mu}^{\prime}(\mathfrak{m}) \neq \boldsymbol{N}$, we have $\boldsymbol{\Psi}_{Y}(\mathfrak{m}) \neq \boldsymbol{N}$, implying that $Y$ is singular with respect to $\boldsymbol{\Psi}$.

Accordingly, in Proposition 16 and in the discussion after it, we may allow to put $U=\mathfrak{a}+\mathfrak{m}_{2}$ and $V=\mathfrak{m}_{1}$. Therefore, by Proposition 16 and Lemma 21, we get (1) of Theorem 11. Further, putting $Y_{0}=Y_{2} \in \mathfrak{m}_{2}$, $\xi^{0}=\mu$ and $\eta=Y_{1}$ into (5.6), we get (2) of Theorem 11. The assertion (3) of Theorem 11 follows from Lemma 17 (2) and Lemma 21. This completes the proof of the theorem.

\section{Proof of Theorem 10}

Let $\left\{E_{i}(1 \leq i \leq 8)\right\}$ be an orthonormal basis of $\mathfrak{m}_{1}$. (Note that $\operatorname{dim} \mathfrak{m}_{1}=8$.) Let $\boldsymbol{\Psi} \in \mathcal{G}_{o}(\boldsymbol{N})$ and let $\mathbf{A}, \mathbf{B}$ be the vectors of $\boldsymbol{N}$ stated in Theorem 11. We define vectors $\left\{\mathbf{F}_{i}(1 \leq i \leq 10)\right\}$ of $\boldsymbol{N}$ by setting $\mathbf{F}_{i}=$ $\boldsymbol{\Psi}\left(\mu, E_{i}\right) /(\mu, \mu)(1 \leq i \leq 8), \mathbf{F}_{9}=(\mathbf{A}+\mathbf{B}) / 2 \sqrt{3}|\mu|$ and $\mathbf{F}_{10}=(\mathbf{A}-\mathbf{B}) / 2|\mu|$. We now show that $\left\{\mathbf{F}_{i}(1 \leq i \leq 10)\right\}$ forms an orthonormal basis of $\boldsymbol{N}$. By Theorem 11 (3) we have $\left\langle\mathbf{F}_{i}, \mathbf{F}_{j}\right\rangle=\delta_{i j}(1 \leq i, j \leq 8)$, where $\delta_{i j}$ denotes Kronecker's delta. Moreover, since $\left\langle\mathbf{A}, \mathbf{F}_{i}\right\rangle=\left\langle\mathbf{B}, \mathbf{F}_{i}\right\rangle=0(1 \leq i \leq 8)$ (see Theorem $11(1 d)$ ), we have $\left\langle\mathbf{F}_{9}, \mathbf{F}_{i}\right\rangle=\left\langle\mathbf{F}_{10}, \mathbf{F}_{i}\right\rangle=0(1 \leq i \leq 8)$. The equalities $\left\langle\mathbf{F}_{9}, \mathbf{F}_{9}\right\rangle=\left\langle\mathbf{F}_{10}, \mathbf{F}_{10}\right\rangle=1$ and $\left\langle\mathbf{F}_{9}, \mathbf{F}_{10}\right\rangle=0$ immediately follow from Theorem $11(1 a)$.

Now let $\boldsymbol{\Psi}^{\prime}$ be another element of $\mathcal{G}_{o}(\boldsymbol{N})$. Let $\mathbf{A}^{\prime}$ and $\mathbf{B}^{\prime}$ be the vectors stated in Theorem 11 for $\boldsymbol{\Psi}^{\prime}$. As in the case of $\boldsymbol{\Psi}$ we can also define an orthonormal basis $\left\{\mathbf{F}_{i}^{\prime}(1 \leq i \leq 10)\right\}$ of $\boldsymbol{N}$. Then, there is an element $h \in$ $O(10)$ satisfying $\mathbf{F}_{i}^{\prime}=h \mathbf{F}_{i}(1 \leq i \leq 10)$. Here we note that $\mathbf{A}^{\prime}=h \mathbf{A}, \mathbf{B}^{\prime}=$ $h \mathbf{B}$ and $\boldsymbol{\Psi}^{\prime}\left(\mu, E_{i}\right)=h \boldsymbol{\Psi}\left(\mu, E_{i}\right)(1 \leq i \leq 8)$. Set $\boldsymbol{\Phi}=\boldsymbol{\Psi}^{\prime}-h \boldsymbol{\Psi} \in S^{2} \mathfrak{m}^{*} \otimes \boldsymbol{N}$. Then, by Theorem 11 (1) we have

$$
\boldsymbol{\Phi}\left(\mathfrak{a}+\mathfrak{m}_{2}, \mathfrak{a}+\mathfrak{m}_{2}\right)=\boldsymbol{\Phi}\left(\mathfrak{m}_{1}, \mathfrak{m}_{1}\right)=\boldsymbol{\Phi}\left(\mathfrak{a}, \mathfrak{m}_{1}\right)=0 .
$$

By the fact $\left[\left[\mu, \mathfrak{m}_{1}\right], \mathfrak{m}_{2}\right] \subset \mathfrak{m}_{1}$ and Theorem 11 (2), we have

$$
\boldsymbol{\Phi}\left(\mathfrak{m}_{2}, \mathfrak{m}_{1}\right) \subset \boldsymbol{\Phi}\left(\mu,\left[\left[\mu, \mathfrak{m}_{1}\right], \mathfrak{m}_{2}\right]\right) \subset \boldsymbol{\Phi}\left(\mathfrak{a}, \mathfrak{m}_{1}\right)=0,
$$

which proves that $\mathbf{\Phi}\left(\mathfrak{m}_{2}, \mathfrak{m}_{1}\right)=0$. Therefore, we have $\mathbf{\Phi}=0$, i.e., $\boldsymbol{\Psi}^{\prime}=h \boldsymbol{\Psi}$. This implies that the Gaussian variety $\mathcal{G}_{o}(\boldsymbol{N})$ is EOS. This completes the 
proof of Theorem 10.

\section{References}

[1] Agaoka Y., Isometric immersions of SO(5). J. Math. Kyoto Univ. 24 (1984), 713724 .

[2] Agaoka Y. and Kaneda E., On local isometric immersions of Riemannian symmetric spaces. Tôhoku Math. J. 36 (1984), 107-140.

[ 3 ] Agaoka Y. and Kaneda E., An estimate on the codimension of local isometric imbeddings of compact Lie groups. Hiroshima Math. J. 24 (1994), 77-110.

[4] Agaoka Y. and Kaneda E., Local isometric imbeddings of symplectic groups. Geometriae Dedicata 71 (1998), 75-82.

[5] Agaoka Y. and Kaneda E., Strongly orthogonal subsets in root systems. Hokkaido Math. J. 31 (2002), 107-136.

[6] Agaoka Y. and Kaneda E., A lower bound for the curvature invariant $p(G / K)$ associated with a Riemannian symmetric space $G / K$. Hokkaido Math. J. 33 (2004), $153-184$

[7] Agaoka Y. and Kaneda E., Local isometric imbeddings of $P^{2}(\boldsymbol{H})$ and $P^{2}(\boldsymbol{C a y})$. Hokkaido Math. J. 33 (2004), 399-412.

[8] Agaoka Y. and Kaneda E., Local isometric imbeddings of Grassmann manifolds. in preparation.

[9] Allendoerfer C.B., Rigidity for spaces of class greater than one. Amer. J. Math. 61 (1939), 633-644.

[10] Bishop R.L. and Crittenden R.J., Geometry of Manifolds. Academic Press, New York, 1964.

[11] Cartan E., Sur la possibilité de plonger un espace riemannien donné dans un espace euclidien. Ann. Soc. Pol. Math. 6 (1927), 1-7.

[12] Helgason S., Differential Geometry, Lie Groups, and Symmetric Spaces. Academic Press, New York, 1978.

[13] Kaneda E., On local isometric immersions of the spaces of negative constant curvature into the euclidean spaces. J. Math. Kyoto Univ. 19 (1979), 269-284.

[14] Kaneda E., On the Gauss-Codazzi equations. Hokkaido Math. J. 19 (1990), 189-213.

[15] Kaneda E., Types of the canonical isometric imbeddings of symmetric R-spaces. Hokkaido Math. J. 22 (1993), 35-61.

[16] Kaneda E. and Tanaka N., Rigidity for isometric imbeddings. J. Math. Kyoto Univ. 18 (1978), 1-70.

[17] Kobayashi S., Isometric imbeddings of compact symmetric spaces. Tôhoku Math. J. 20 (1968), 21-25.

[18] Kobayashi S. and Nomizu K., Foundations of Differential Geometry II. Interscience Publishers, New York, 1969.

[19] Nomizu K., Uniqueness of the normal connections and congruence of isometric immersions. Tôhoku Math. J. 28 (1976), 613-617. 
[20] Ôtsuki T., Isometric imbedding of Riemann manifolds in a Riemann manifold. J. Math. Soc. Japan 6 (1954), 221-234.

[21] Szczarba R.H., On existence and rigidity of isometric immersions. Bull. Amer. Math. Soc. 75 (1969), 783-787.

[22] Szczarba R.H., On isometric immersions of Riemannian manifolds in euclidean space. Bol. Soc. Brasil. Mat. 1 (2) (1970), 31-45.

[23] Tanaka N., Rigidity for elliptic isometric imbeddings. Nagoya Math. J. 51 (1973), 137-160.

\section{Y. Agaoka}

Faculty of Integrated Arts and Sciences Hiroshima University

1-7-1 Kagamiyama, Higashi-Hiroshima City

Hiroshima, 739-8521, Japan

E-mail: agaoka@mis.hiroshima-u.ac.jp

E. Kaneda

Department of International Studies Osaka University of Foreign Studies 8-1-1 Aomadani-Higashi, Minoo City Osaka, 562-8558, Japan

E-mail: kaneda@osaka-gaidai.ac.jp 